\title{
Experimental Verification of Solid-like and Fluid-like States in the Homogeneous Fluidization Regime of Geldart A Particles
}

DOI:

10.1021/acs.iecr.7b04559

\section{Document Version}

Accepted author manuscript

Link to publication record in Manchester Research Explorer

Citation for published version (APA):

Guo, Q., Meng, S., Zhao, Y., Ma, L., Wang, D., Ye, M., Yang, W., \& Liu, Z. (2018). Experimental Verification of Solid-like and Fluid-like States in the Homogeneous Fluidization Regime of Geldart A Particles. Industrial and Engineering Chemistry Research, 57(7), 2670-2686. https://doi.org/10.1021/acs.iecr.7b04559

Published in:

Industrial and Engineering Chemistry Research

\section{Citing this paper}

Please note that where the full-text provided on Manchester Research Explorer is the Author Accepted Manuscript or Proof version this may differ from the final Published version. If citing, it is advised that you check and use the publisher's definitive version.

\section{General rights}

Copyright and moral rights for the publications made accessible in the Research Explorer are retained by the authors and/or other copyright owners and it is a condition of accessing publications that users recognise and abide by the legal requirements associated with these rights.

\section{Takedown policy}

If you believe that this document breaches copyright please refer to the University of Manchester's Takedown Procedures [http://man.ac.uk/04Y6Bo] or contact uml.scholarlycommunications@manchester.ac.uk providing relevant details, so we can investigate your claim.

\section{OPEN ACCESS}


Experimental verification of solid-like and fluid-like states in the homogeneous fluidization regime of Geldart A particles

\title{
Qiang Guo ${ }^{\mathrm{a}, \mathrm{b}}$, Shuanghe Meng ${ }^{\mathrm{a}}$, Yinfeng Zhao ${ }^{\mathrm{a}}$, Likun Ma ${ }^{\mathrm{a}, \mathrm{b}}$, Dehu Wanga, Mao Yea,*, Wuqiang Yangc, Zhongmin Liu ${ }^{a}$
}

a Dalian National Laboratory for Clean Energy and National Engineering Laboratory for MTO, Dalian Institute of Chemical Physics, Chinese Academy of Sciences, Dalian 116023, China

${ }^{\mathrm{b}}$ University of Chinese Academy of Sciences, Beijing 100049, China

${ }^{c}$ School of Electrical and Electronic Engineering, The University of Manchester, Manchester M13 9PL, United Kingdom

* Corresponding author: Mao Ye, email: maoye@ dicp.ac.cn

\begin{abstract}
The mechanisms underlying homogeneous fluidization of Geldart A particles have been debated for decades. Some ascribed the stability to inter-particle forces, while others insisted a purely hydrodynamic explanation. Valverde et al. (2001) fluidized 8.53- $\mu \mathrm{m}$ (i.e., Geldart C) particles by the addition of fumed silica nanoparticles and found that even during homogeneous fluidization both solid-like and fluid-like behavior can be distinguished. However, it is still unclear whether both states exist for true Geldart A particles. In this paper, particulate fluidization characteristics of three typical Geldart A powders were studied by camera recording, electrical capacitance tomography, and pressure fluctuation. For the first time, the existence of both solid-
\end{abstract}


like state dominated by inter-particle forces and fluid-like state by fluid dynamics during homogeneous expansion of Geldart A particles was experimentally verified. Furthermore, the ability and performance of the used measurement techniques to identify different flow regimes were compared.

Keywords: Geldart A particles; homogeneous fluidization; electrical capacitance tomography; pressure fluctuation; solid-like; fluid-like

\section{Introduction}

Gas-solid fluidization occurs when a bed of particles is suspended by an upward gas flow that exceeds the minimum fluidization velocity $\left(u_{m f}\right)$ required to balance the weight of particles. Fluidization behavior depends much on the physical properties of particles employed. Geldart ${ }^{1}$ suggested a classification diagram for particles fluidized in air under atmospheric conditions based on the mean particle size and density difference between particles and surrounding fluidization medium. This diagram has later become a standard to demarcate the behavior of gas-solid fluidization. ${ }^{2}$ According to Geldart's classification, particles of size less than $30 \mu \mathrm{m}$ belong to Group C and fluidize poorly due to strong cohesive forces compared to their gravity. Conversely, Group D particles have the biggest size typically larger than $800 \mu \mathrm{m}$, and the fluidization of Group D particles often demonstrates a spouting behavior. Particles of intermediate size are classified into two groups according to whether bed bubbles or not when the superficial gas velocity $\left(u_{g}\right)$ is larger than $u_{m f}$. For Group B particles that have the size between $100 \mu \mathrm{m}$ and $800 \mu \mathrm{m}$, bed bubbles immediately after $u_{m f}$. While for Group A particles, the size is between $30 \mu \mathrm{m}$ and $100 \mu \mathrm{m}$, and 
there is an interval of non-bubbling expansion regime (also called homogeneous fluidization or particulate fluidization) between $u_{m f}$ and the minimum bubbling velocity $\left(u_{m b}\right)$, in which no macroscopic bubbles appear.

Although homogeneous fluidization of Geldart A particles has received considerable attention for a long time, ${ }^{3-12}$ it is still debated whether it originates from inter-particle forces or hydrodynamic factors. ${ }^{13}$ Menon and Durian $^{8}$ found that particle velocity fluctuations are essentially zero during the interval of homogeneous fluidization, which led them to conclude that enduring contacts of particles make particles stay at rest and thus the bed keeps solid-like. Rietema ${ }^{5}$ demonstrated that inter-particle forces like cohesion and friction can give an effective elastic modulus to keep the bed surface stable rather than sliding off when a homogeneous fluidized bed is tilted. On the other hand, the inter-particle force explanation was argued by researchers who tended to attribute the stability to the effect of pure fluid dynamics. Cody et al. ${ }^{9}$ measured granular temperature, which is a metric of velocity fluctuation of particles, using an acoustic shot noise probe, and found that once $u_{m f}$ is reached, the average granular temperature increases with the increase in $u_{g}$, showing a fluid-like behavior. From a theoretical viewpoint, Garg and Pritchett ${ }^{6}$ reported that the stability of homogeneous fluidization can be well predicted by adding a fluid dynamic force to the linear momentum balance equation of solid phase. Similarly, Foscolo and Gibilaro $^{4}$ claimed that a theoretical criterion proposed based on the interaction between particles and fluid enables a full prediction of the onset of bubbling in fluidized beds.

Valverde et al. ${ }^{14-16}$ were pioneers who tried to clarify the debate by experiments. By adding fumed silica nanoparticles, they fluidized a commercially available xerographic toner with the 
volume average diameter of $8.53 \mu \mathrm{m},{ }^{14}$ which actually belongs to Geldart C powder ${ }^{1}$ (see later in Figure 1d). Surprisingly, their results showed that because of the reduced cohesion between grains by the added fumed silica nanoparticles, the system exhibits a considerably wide interval of homogeneous expansion with the ratio of $u_{m b}$ to $u_{m f}$ about 40 , which is much higher than that of the normally used Geldart A particles, say $1 \sim 3 .{ }^{17}$ Within such a wide interval, they investigated the bed structure in detail and found that both solid-like and fluid-like states exist in the homogeneous fluidization regime, indicating that the stability of homogeneous fluidization may have two distinct origins: in the solid-like state, inter-particle forces dominate; while in the fluid-like state, hydrodynamic factors dominate. Later, the same research group ${ }^{15}$ explored the effect of particle size on the extension of the fluid-like state using four sets of nanoparticles-coated powders with the sizes of $7.8 \mu \mathrm{m}, 11.8 \mu \mathrm{m}, 15.4 \mu \mathrm{m}$, and $19.1 \mu \mathrm{m}$, showing that the fluid-like state shortens as the particle size increases. An extrapolation of their results indicates that there would be only solidlike state in the homogeneous expansion regime for particles with the size larger than $40 \mu \mathrm{m}$. However, it is evident that these conclusions were drawn based on the modified Geldart C particles. To our best knowledge, there is yet no experimental report on whether both solid-like and fluidlike states exist for true Geldart A particles. ${ }^{18}$ For example, a recent work ${ }^{19}$ found that a magnetic field can extend the stable fluidization interval of a magnetic powder with the average size of 26.2 $\mu \mathrm{m}$, which is on the boundary of Geldart A and Geldart C particles, but still the bed bubbles just after the solid-like state.

It should be stressed that, however, fluidization behaviors of the modified Geldart $\mathrm{C}$ particles and true Geldart A particles are different from each other. In addition to some apparent fluidization 
characteristics such as the expansion ratio, maximum free volume, and ratio of $u_{m b}$ to $u_{m f}$, the modified Geldart C particles undergo a dynamic aggregation process, ${ }^{16,20-23}$ while for Geldart A particles, it is still questioned whether or not aggregates present in the homogeneous expansion region. ${ }^{24-26}$ Homogeneous fluidization of Geldart A particles is of great relevance: for fundamental research, it is closely associated with the instability and origin of mesoscale structures in fluidized beds ${ }^{3}$ for industrial application, the optimal operation for particles circulation in fluidized bed reactor-regenerator systems, such as in fluid catalytic cracking (FCC) and methanol to olefins (MTO) processes, is achieved when particles flow downwards a standpipe in a bubble-free condition, ${ }^{27}$ which is actually homogeneous fluidization. Furthermore, homogeneous fluidization features naturally for Geldart A particles, but not for Geldart C particles. Therefore, it is significant to investigate whether both solid-like and fluid-like states exist in the homogeneous expansion regime for true Geldart A particles as well.

In fact, before the experimental works by Valverde et al., ${ }^{14-16}$ Fortes et al. ${ }^{10}$ suggested a similar distinct-origin mechanism using a two-fluid model for particles with the $u_{m f}$ of $2.4 \mathrm{~mm} / \mathrm{s}$ (note that this value is close to the $u_{m f}$ of the particles used in this work), which should be categorized to Group A. Ye et al. ${ }^{11,12}$ performed numerical simulations using discrete particle model (DPM) for homogeneous fluidization of Geldart A particles, and found that some typical features in the homogeneous fluidization regime such as homogeneous bed expansion and gross particle circulation can be simulated. Although the existence of both solid-like and fluid-like states was not mentioned in their works, it is clearly noted that the particle velocity fluctuations at the former three points in Figure 11 given by Ye et al. ${ }^{11}$ and the former four points in Figure 3 given by Ye et 
al. ${ }^{12}$ are definitely different from other points in the homogeneous expansion regime, and seem to behave like those in the fixed bed. These simulation works imply that both solid-like and fluidlike states may also exist in the homogeneous expansion regime for Geldart A particles, which, however, needs further experimental verification.

In this work, particulate fluidization characteristics of three typical Geldart A powders were carefully investigated by three widely used measurement techniques, i.e., camera recording, electrical capacitance tomography (ECT), and pressure fluctuation. The purpose of the work is to validate whether both solid-like and fluid-like states exist in the homogeneous expansion regime for Geldart A particles by experiments. The organization of this article is as follows. First, experimental setup, measurement techniques, and experimental methods are briefly introduced. Then, measurement results from different techniques associated with the corresponding discussion are presented, followed by a short comparison on the ability and performance of the used three measurement techniques to identify different flow regimes. Subsequently, different fluidization behaviors between true Geldart A particles and modified Geldart C particles are compared. Finally, the paper ends with some conclusions.

\section{Experimental setup and methods}

\subsection{Experimental setup and measurement techniques}

All experiments were carried out in a cylindrical fluidized bed with a twin-plane ECT sensor, a differential pressure transducer, and a camera, as shown in Figure 1a. The fluidized bed was a quartz glass column with the height of $1 \mathrm{~m}$ and inner diameter of $5 \mathrm{~cm}$. Air flow under ambient 
conditions was distributed to the bottom of the column using an expanded polystyrene foam with the average pore size of $10 \mu \mathrm{m}$. Preliminary tests revealed that such a distributor can induce a uniform and stable gas flow across the cross-section of the bed. The flow rate of air was precisely controlled by a Brooks mass flow controller (Brooks SLA5800 Series).

[Figure 1. (a) Experimental setup. (b) ECT sensor structure. (c) Particle size distribution of the powders. (d) Situation of the powders in the Geldart diagram.]

The ECT sensor consisted of two planes to obtain the cross-sectional overall solid concentration at two different heights. The mid-position of the lower and upper planes located at $11.25 \mathrm{~cm}$ and $26.25 \mathrm{~cm}$ above the distributor, respectively. Both planes had the same structure, as shown in Figure 1b, with eight electrodes being stuck to the outside wall of the bed and covered by an earthed screen to eliminate external interference. An AC-based ECT system ${ }^{28}$ with 16 channels was used to measure the inter-electrode capacitance. Because the length of each electrode was $2.5 \mathrm{~cm}$, the obtained overall solid concentration is an average over the measurement region encompassed by the vertical height of the electrodes.

The differential pressure transducer was used to measure the bed pressure drop and its fluctuation. To prevent disturbance to the flow, the positive and negative sides of the transducer were connected to taps below the distributor and at the top end of the freeboard, respectively. The pressure drop across the distributor was first determined as a function of $u_{g}$ with the tube empty. Then, the pressure drop across the bed itself was obtained by subtracting the distributor pressure 
drop from the measured total pressure drop. ${ }^{7}$

To enable visual observation of fluidization behavior, an Olympus i-speed 3 camera was also used. In each measurement, the lens of the camera was always adjusted to be focused on the bed surface. Along the front side of the column, a paper ruler was taped to measure the bed height.

\subsection{Measurement of the overall solid concentration using ECT}

The measurement principle of ECT is to reconstruct permittivity distribution as a presentation of material distribution inside the sensing area based on the measured inter-electrode capacitance and a specific image reconstruction algorithm. Successful application of ECT strongly depends on the employed algorithm. Yang and Peng ${ }^{29}$ have evaluated a variety of algorithms including singlestep and iterative ones, and suggested that Landweber iteration technique with a projection can

provide the best results for most cases, which was further confirmed by Guo et al. ${ }^{30}$ The formula of the projected Landweber iteration is written as

$$
\begin{gathered}
G_{k}=P\left[G_{k-1}+\alpha S^{T}\left(\lambda-S G_{k-1}\right)\right] \\
P[f(x)]=\left\{\begin{array}{cc}
0 & \text { if } f(x)<0 \\
f(x) & \text { if } 0 \leq f(x) \leq 1 \\
1 & \text { if } f(x)>1
\end{array}\right.
\end{gathered}
$$

where $G$ is a vector indicating the normalized permittivity distribution, i.e., the reconstructed grey level in each image pixel, $S$ is the normalized sensitivity matrix, $S^{T}$ is the transpose of $S, \alpha$ is the step length, and $k$ is the index of iteration steps. A large $\alpha$ can speed up the iteration process, but if it is too large the process will be difficult to converge. In this work, $\alpha$ takes a constant value of 2 following Yang et al. ${ }^{31}$ The projection operator $P$ in the Landweber iteration method is used to constrain the estimated image to ensure $G \in[0,1]$. 
The initial estimation $G_{0}$ in Equation 1 is calculated by linear back projection (LBP) method, which is the simplest single-step algorithm for ECT, as formulated by

$$
G_{0}=\frac{S^{T} \lambda}{S^{T} u_{\lambda}}
$$

where $u_{\lambda}$ is a vector of ones. The division in the LBP method is manipulated in a one-to-one mode, that is to say, one numerator component is divided by the corresponding denominator component. ${ }^{29}$

The parameter $\lambda$ in Equations 1 and 3 is the normalized capacitance vector, and is expressed

as

$$
\lambda=\frac{C_{M}-C_{L}}{C_{H}-C_{L}}
$$

where $C_{M}$ is the measured capacitance vector for an arbitrary distribution, and $C_{L}$ and $C_{H}$ are the capacitance vectors measured in an empty and packed bed, respectively. The procedure to obtain $C_{L}$ (low calibration) and $C_{H}$ (high calibration) is known as system calibration.

Equation 5 is commonly used to calculate the sensitivity matrix:

$$
S_{i j}^{*}(x, y)=-\iint_{p(x, y)} \frac{\nabla \varphi_{i}(x, y)}{V_{i}} \cdot \frac{\nabla \varphi_{j}(x, y)}{V_{j}} d x d y
$$

where $S_{i j}^{*}$ is the sensitivity between the $i-j$ electrode pair at the location of the pixel $p(x, y)$ and $\varphi_{i}(x, y)$ and $\varphi_{j}(x, y)$ are the potential distributions inside the imaging area when the $i$ th and $j$ th electrodes are excited by applying voltages of $V_{i}$ and $V_{j}$, respectively. Thereafter, the normalized sensitivity matrix $S$ is calculated as

$$
S_{m n}=\frac{S_{m n}^{*}}{\sum_{n=1}^{N} S_{m n}^{*}}
$$

where $S_{m n}$ and $S_{m n}^{*}$ are the entries in the $m$ th row and $n$th column of $S$ and $S^{*}$, respectively, and $N$ is the number of pixels in the imaging area. For more detailed information regarding image reconstruction using ECT, interested readers can refer to the work by Guo et al. ${ }^{30}$ 
Although the projected Landweber iteration algorithm can reconstruct satisfied images, the results are still poorer than those recorded directly by a camera due to the "soft-field" of ECT, ${ }^{29,30}$ especially when the bed is in the homogeneous expansion regime, where no macroscopic bubbles appear. In addition, it has been confirmed that the raw capacitance and overall solid concentration signals obtained by ECT are much more sensitive to a small change of flow patterns than images. ${ }^{32,33}$ Therefore, these two quantities, but not the reconstructed images, are used following

previous works. ${ }^{33-35}$ Here, the raw capacitance is the average over all possible electrode pairs and the overall solid concentration $\beta$ is obtained via pixel averaging:

$$
\beta=\theta \cdot \bar{G}=\theta \cdot \frac{\sum_{i=1}^{N} G_{i} s_{i}}{\sum_{i=1}^{N} s_{i}}
$$

where $\theta$ is the packed bed solid concentration, which was estimated as 0.61 for all three studied powders, and $s$ is the area of each image pixel.

\subsection{Materials and experimental procedure}

Three powders, i.e., two kinds of FCC powders and a $\mathrm{Al}_{2} \mathrm{O}_{3}$ powder, were used in this research. Figure 1c shows their particle size distributions and Table 1 summarizes their physical properties, including particle density, Sauter mean diameter, and fine particles content. According to Geldart's classification ${ }^{1}$ as shown in Figure 1d, the fluidized particles used in this work all belong to typical Group A particles.

[Table 1. Physical properties of the powders]

As mentioned before, the ECT sensor needs to be calibrated before measurements can be 
taken. At first, low calibration, in which $C_{L}$ is measured, was done by emptying the fluidized bed.

Then, a known amount of a specific powder was poured into the bed. Next, $u_{g}$ was set to a sufficiently large value to make the bed fluidize in the bubbling regime. When the bed reaches a steady state, $u_{g}$ was decreased to zero slowly, causing the bed to finally a packed state with the static bed height of approximately $38 \mathrm{~cm}$. Then high calibration, in which $C_{H}$ is measured, was performed.

Both fluidization and defluidization experiments were carried out. In each experiment, $u_{g}$ was increased in a fluidization process or decreased in a defluidization process using a stepwise method, and the change in velocity in every adjustment was kept small. At each velocity, runs of 15 minutes duration were conducted. Then, the measurement results were recorded in a 100 seconds period with the data acquisition rates of $60 \mathrm{~Hz}, 120 \mathrm{~Hz}$, and $10 \mathrm{~Hz}$ for ECT, pressure, and camera, respectively. To ensure the consistency of the results, each experiment was repeated three times.

\section{Results and discussion}

\subsection{Visual observations from the camera}

Figure 2 shows an example of four consecutive snapshots at different superficial gas velocities for FCC I powder. More intuitive and informative presentations are shown as videos in Supporting Information Videos S1 and S2, in which a collection of 60 photos recorded by the camera in a time duration of 6 seconds is included at each superficial gas velocity. In Figure 2 and Videos S1 and S2, the tags on the top right of the snapshots indicate the sequence number in the 60 consecutive photos and the corresponding bed height. The bed surface is at the location of 4.5 
$\mathrm{mm}$ in the paper ruler when $u_{g}$ is $0 \mathrm{~mm} / \mathrm{s}$.

As can be seen, with the increase in $u_{g}$, the bed surface exhibits different behavior. Three different flow regimes, i.e., fixed bed, homogeneous fluidization in a conventional sense, and bubbling fluidization can be clearly identified. In the fixed bed regime (see Figure 2a and Video S1a), the bed surface remains stationary at a constant location that is the same as that in the initial packed bed and the topography of the bed surface is also the same for different velocities. Note that the folds on the bed surface are formed in the sedimentation procedure (see section 2.3.). In the homogeneous fluidization regime (see Figures $2 b$ and $2 c$ and Videos $S 1 b$ and $S 2 a$ ), the bed expands gradually with the increase in $u_{g}$, and in each $u_{g}$, the bed height remains stable without any macroscopic bubbles that can burst to cause the bed surface to oscillate. ${ }^{1,13}$ Upon further increasing $u_{g}$, the bed begins to be operated in the bubbling regime, and some characteristic fluidization features in the bubbling regime, such as obvious big bubbles bursting on the bed surface and oscillating bed height, ${ }^{1,11}$ can be easily seen in Figure $2 \mathrm{~d}$ and Video S2b.

[Figure 2. Visual snapshots taken by the camera: (a) $u_{g}=1.27 \mathrm{~mm} / \mathrm{s}$ in the fixed bed regime, (b) $u_{g}=3.18 \mathrm{~mm} / \mathrm{s}$ in the solid-like homogeneous fluidization regime, $(c) u_{g}=5.09 \mathrm{~mm} / \mathrm{s}$ in the uniform non-bubbling fluid-like fluidization regime, and $(d) u_{g}=7.85 \mathrm{~mm} / \mathrm{s}$ in the bubbling fluidization regime. The fluidized powder is FCC I.]

Although both Figures $2 \mathrm{~b}$ and $2 \mathrm{c}$ or Videos $\mathrm{S} 1 \mathrm{~b}$ and $\mathrm{S} 2 \mathrm{a}$ belong to the homogeneous fluidization regime featured with a stable bed height, a careful check suggests that there are still 
differences between them. In Figure $2 b$ and Video $S 1 b$, the bed surface remains static just like that in the fixed bed regime and the topography of the bed surface remains invariant for a certain velocity. In fact, not only on the bed surface, but also at other locations, no apparent particle movement can be seen. While in Figure 2c and Video S2a, the bed surface is no longer static. By contrast, some dynamic undulations and small bubbles are shown on the bed surface. These small bubbles are not obvious bubbles that have the diameter of $5 \mathrm{~mm}$ as recognized as the beginning of the bubbling regime by Geldart, ${ }^{1}$ because they cannot make the bed height oscillate, which is a characteristic feature in the bubbling regime. Instead, these small bubbles are more like voids and cavities in literature, ${ }^{16,36}$ and will be named as microscopic bubbles to be distinguished from the macroscopic bubbles in the bubbling regime. Note that the size of the bed used by Geldart ${ }^{1}$, say 5 $\mathrm{cm}$, is the same as that used in this work. Moreover, particle movement within a small range can also be seen at other locations of the bed in this regime.

Overall, the snapshots shown in Figure $2 \mathrm{~b}$ and Video S1b display a solid-like behavior, in which the bed remains static, while those in Figure 2c and Video S2a show a fluid-like behavior, in which microscopic bubbles and particle movement can be seen. It can be then concluded from these observations that both solid-like and fluid-like states exist in the homogeneous fluidization regime for FCC I powder.

The above findings are based solely on the visual observations from the camera. However, the application of the visual observation method is known to be limited. Therefore, the exploration using other measurement techniques is necessary. The following will show the fluidization characteristics measured by ECT and pressure fluctuation methods. Note that, as seen from the 
visual observations and previous works by Valverde et al. ${ }^{16}$, the apparently homogenous fluid-like state is characterized by the presence of microscopic bubbles. Therefore, the solid-like state will be named as "solid-like homogeneous fluidization regime", while the fluid-like state will be named as "uniform non-bubbling fluid-like fluidization regime" in the following text.

\subsection{ECT measurement results}

Time and frequency domain analysis of the overall solid concentration can be used to identify fluidization regime and its transition in the fluidized beds. ${ }^{37-40}$ In this section, the overall solid concentration is analyzed on the basis of standard deviation ( $\mathrm{Sd}$ ) and power spectral density.

[Figure 3. Sampled signals of the overall solid concentration at ECT upper plane for different flow regimes: (a) $u_{g}=1.91 \mathrm{~mm} / \mathrm{s}$ in the fixed bed regime, (b) $u_{g}=3.40 \mathrm{~mm} / \mathrm{s}$ in the solid-like homogeneous fluidization regime, (c) $u_{g}=5.31 \mathrm{~mm} / \mathrm{s}$ in the uniform non-bubbling fluid-like fluidization regime, and $(d) u_{g}=7.85 \mathrm{~mm} / \mathrm{s}$ in the bubbling fluidization regime. The fluidized powder is FCC I.]

Figure 3 shows the transient overall solid concentration signals at ECT upper plane for the fixed bed, solid-like homogeneous fluidization, uniform non-bubbling fluid-like fluidization, and bubbling bed regimes. The ordinate range of Figures $3 a, 3 b$, and $3 c$ is set to be the same, say 0.01 . It can be seen that the overall solid concentration varies significantly in amplitude for different flow regimes. Obviously, the fluctuation of the overall solid concentration increases with the evolution of fluidization regime, and more specifically no marked fluctuation is observed in the 
fixed bed and solid-like homogeneous fluidization regimes, while a small but clearly recognizable fluctuation is seen in the uniform non-bubbling fluid-like fluidization regime, and the fluctuation becomes more significant when the bed is in the bubbling regime.

Figure 4 shows the average and $\mathrm{Sd}$ of the overall solid concentration as a function of $u_{g}$ in a fluidization process for both ECT lower and upper planes. The repeated experimental results in three separate measurements at ECT upper plane are shown in Figures 5a and 5b to show the consistency of the results. As can be seen, there are two clear turning points occurring for the average concentration with increasing $u_{g}$. In a recent paper, ${ }^{41}$ it has been verified that the first turning point corresponds to $u_{m f}$. Because in the fixed bed regime, the constant bed height results in an unchanged solid concentration, and when $u_{m f}$ is reached, the solid concentration starts to decrease monotonously as a result of bed expansion in the homogeneous fluidization regime. The second turning point is inferred to refer to the so-called bed contraction phenomenon, ${ }^{42,43}$ because for Geldart A particles, the bed expands gradually in the homogeneous fluidization regime, and then expands to the maximal height in the vicinity of $u_{m b}$, after which the bed collapses due to part of gas rises in the form of macroscopic bubbles, leading to the increase in the average concentration. It is also important to highlight that in both the solid-like homogeneous and uniform non-bubbling fluid-like fluidization regimes, the average of the overall solid concentration at ECT lower and upper planes almost coincide with each other, confirming that the bed in these two states is truly uniform. When $u_{g}$ is further increased to the bubbling regime, a heterogeneity of the overall solid concentration along the axial direction of the fluidized bed is presented, and more specifically the concentration at upper plane is higher than that at lower plane, which seems inconsistent with 
previous conclusion ${ }^{44}$ that bubble size increases along the bed height and so that the overall solid concentration decreases with the height. However, Figures 5, 7, and 11 in a recent experimental and computational study by Liu et al. ${ }^{45}$ also show a similar trend of the axial profile of solid concentration in a bubbling fluidized bed with Geldart A particles. These observations can be well explained by the compromise between the bubble growth in the bottom zone and bed collapse in the upper zone of the bed when $u_{g}$ is relatively low (in Figure 4, the maximum of $u_{g}$ is $9.76 \mathrm{~mm} / \mathrm{s}$ ). Indeed, it was found that when $u_{g}$ exceeds approximately 3 times the $u_{m b}$, the overall solid concentration at lower plane is higher than that at upper plane (not shown).

[Figure 4. Average and Sd of the overall solid concentration at both ECT lower and upper planes against $u_{g}$. The fluidized powder is FCC I.]

For Sd of the overall solid concentration, it is obvious from Figure 4 that there are three plateaus (see the tag in Figure 4), in which Sd only shows a little change against $u_{g}$ in each plateau. According to these three plateaus, the curve is divided into four sections as shown in Figure 4. It is demonstrated by the videos taken in each section as shown in Supporting Information that these four sections correspond to the fixed bed, solid-like homogeneous fluidization, uniform nonbubbling fluid-like fluidization, and bubbling bed regimes, respectively.

In the fixed bed regime, the bed surface is at the location of $4.5 \mathrm{~mm}$ in the paper ruler, and Sd of the overall solid concentration is a little higher than zero due to measurement noise. Then in the solid-like homogeneous fluidization state, although the bed expands with the increase in $u_{g}$, the 
bed surface remains static like that in the fixed bed regime, and Sd increases a little, possibly because of the enlarged noise (see later in Figure 6) associated with electrostatic effects generated by particle-particle friction. ${ }^{46}$ When $u_{g}$ is further increased to the uniform non-bubbling fluid-like fluidization regime, the bed continues to expand with a stable bed height. But because of the appearance of microscopic bubbles and particle movement as shown in Figure 2c and Video S2a, Sd shows an increase compared to the solid-like homogeneous fluidization state. For the same reason, a small fluctuation of $\mathrm{Sd}$ is shown in the third plateau for the uniform non-bubbling fluidlike fluidization regime, while Sd is nearly constant in the first two plateaus for the fixed bed and solid-like homogeneous fluidization regimes. When the bed is operated in the bubbling regime, the bed height begins to oscillate. As a result, Sd of the overall solid concentration also increases sharply. Especially, $\mathrm{Sd}$ in the bubbling regime is ten times more than that in other three regimes.

In Figure 4, labels indicating $u_{m f}, u_{m b}$, and the transition velocity between the solid-like homogeneous and uniform non-bubbling fluid-like fluidization regimes $u_{c}{ }^{14}$ are marked. Note that here, $u_{m f}, u_{c}$, and $u_{m b}$ are identified from the terminal point of each plateau in the plot of the Sd of the overall solid concentration against $u_{g}$. In fact, it was found that the ECT experimental results show good consistency with those obtained from visual observations: the obtained $u_{m f}$ in this way is identical to that from pressure drop measurements (to be discussed later) and visual observations, at which the bed begins to expand; the obtained $u_{c}$ is the same as that from visual observations, at which microscopic bubbles begin to appear on the bed surface; and the obtained $u_{m b}$ also equals to that from visual observations, at which the bed height begins to oscillate and the first clearly recognizable bubble with the size larger than $5 \mathrm{~mm}$ on the bed surface is captured. ${ }^{1}$ The 
experimental values of $u_{m f}$ and $u_{m b}$ for FCC I powder are $2.55 \mathrm{~mm} / \mathrm{s}$ and $7.00 \mathrm{~mm} / \mathrm{s}$, respectively, which are very close to the predicted values of $2.57 \mathrm{~mm} / \mathrm{s}$ and $7.08 \mathrm{~mm} / \mathrm{s}$ by the empirical correlation of Abrahamsen and Geldart, ${ }^{17}$ as expressed in Equations 8 and 9:

$$
\begin{gathered}
u_{m f, c}=0.0009 \times \frac{\left[\left(\rho_{p}-\rho_{g}\right) g\right]^{0.934} d_{p}{ }^{1.8}}{\mu^{0.87} \rho_{g}{ }^{0.066}} \\
u_{m b, c}=2.07 \times \frac{d_{p} \rho_{g}{ }^{0.06}}{\mu_{g}{ }^{0.347}} \times E X P\left(0.716 \times F_{45}\right)
\end{gathered}
$$

where $\rho_{g}$ and $\mu$ are the density and viscosity of air under ambient conditions, respectively, $g$ is the gravitational acceleration, $F_{45}$ is the fraction of fines with the size less than $45 \mu \mathrm{m}$, and $u_{m f}$ and $u_{m b}$ with the subscript $c$ mean the values calculated from the empirical correlation. It is also noted from Figure 4 that the second turning point in the curve of the average solid concentration for both ECT lower and upper planes, which corresponds to the bed contraction phenomenon, is a little higher than the marked $u_{m b}$. Similar results can be found in the DPM simulations by Wang et al. ${ }^{42}$

[Figure 5. Repeated experimental results in three separate measurements for FCC I powder: (a) and $(b)$ are ECT measurement results; $(c)$ and $(d)$ are pressure fluctuation results.]

To exclude the effect of the used Landweber iteration algorithm on the identification of different flow regimes based on the calculated overall solid concentration, the average and noiseto-signal ratio (NSR) of the raw capacitance at ECT upper plane as a function of $u_{g}$ are plotted in Figure 6. The noise-to-signal ratio here is defined as the reciprocal of the signal-to-noise ratio (SNR), which is usually used to indicate the original signal quality of ECT. ${ }^{28-30}$ SNR and NSR are formulated, respectively, by Equations 10 and 11: 


$$
\begin{gathered}
\mathrm{SNR}=20 \times \log _{10} \sqrt{\frac{\sum_{i=1}^{K} C_{i}{ }^{2}}{\sum_{i=1}^{K}\left(C_{i}-\bar{C}\right)^{2}}} \\
\mathrm{NSR}=\frac{1}{\mathrm{SNR}}
\end{gathered}
$$

where $C_{i}$ is the capacitance averaged over all possible inter-electrode in a frame and $\bar{C}$ is the average of $C_{i}$ over all frames $(K)$ measured in a certain velocity. As can be seen, the profiles of the average and NSR of the raw capacitance agree well with the average and Sd of the overall solid concentration, respectively. Therefore, time domain analysis of both the overall solid concentration and raw capacitance can be used to characterize fluidization regime transition in fluidized beds. ${ }^{33-}$ ${ }^{35}$ From Figure 6, it is noted that the NSR is very stable in the fixed bed and solid-like homogeneous fluidization regimes, indicating that the fluctuation of the raw capacitance or overall solid concentration in these two regimes is only due to measurement noise. Especially, the SNR is calculated to be as high as $66 \mathrm{~dB}$ in the fixed bed regime, showing the robust performance of the used AC-based ECT measurement system.

[Figure 6. Average and NSR of the raw capacitance at ECT upper plane against $u_{g}$. The fluidized powder is FCC I.]

Frequency domain analysis can supplement the information obtained from time domain analysis, such as the standard deviation. In Figure 7, the power spectral density of the overall solid concentration for different flow regimes calculated using fast Fourier transformation (FFT) is presented. According to the above analysis, the bed remains static in both the fixed bed and solidlike homogeneous fluidization regimes, and the fluctuation of the overall solid concentration in 
these two states is only due to measurement noise (see Figure 6). As seen in Figures 7a and 7b, indeed, there is no a clear dominant frequency, and the peaks at the locations around $1.5 \mathrm{~Hz}, 3 \mathrm{~Hz}$, $6 \mathrm{~Hz}$, and $9 \mathrm{~Hz}$ are linked to the electronic components in the measurement system. ${ }^{28}$ While in the uniform non-bubbling fluid-like and bubbling fluidization regimes, the dominant frequency at approximately $0.02 \mathrm{~Hz}$ and $0.09 \mathrm{~Hz}$ is clearly seen in Figures 7c and 7d, respectively. Note that in Figures $7 \mathrm{c}$ and $7 \mathrm{~d}$, there are also small peaks at the locations intrinsically derived from the measurement system, however, they are severely suppressed by the dominant frequency that carries the most energy among all frequencies in the spectrum. The dominant frequency in the bubbling regime obtained from ECT results here, $0.09 \mathrm{~Hz}$, is much lower than the typical frequency reported in previous researches, ${ }^{47}$ say about $2 \mathrm{~Hz}$. However, it must be pointed out that to date, little work has been carried out on the FFT analysis at the velocity close to $u_{m b}$ like what has been done in this research, and the studied $u_{g}$ in previous studies ${ }^{33,38,40,47,48}$ are much larger than that used here. As can be seen in Video S2b, indeed, the bubbles appear on the bed surface at a low frequency. The relatively low dominant frequency in the bubbling regime here may due to the compromise between the bubble growth and bed collapse at a relatively low velocity, which has been shown in Figure 4. As expected, when $u_{g}$ is further increased to about 3 times the $u_{m b}$, at which the bubble growth along the bed height plays the leading role, the dominant frequency shifts to approximately $2 \mathrm{~Hz}$ (see Figure 8). It is also noted from Figure 7 that although the dominant frequency in the bubbling fluidization regime is a little higher than that in the uniform nonbubbling fluid-like fluidization regime, the distribution of the power spectral density shows similar pattern between these two regimes. The similarity may come from the fact that the fluctuation 
frequency of the void and cavity structures (microscopic bubbles) in the uniform non-bubbling fluid-like fluidization regime is like that of the macroscopic bubbles in the bubbling fluidization regime for $u_{g}$ above the $u_{m b}$ and below about 3 times the $u_{m b}$, and further confirms that the uniform non-bubbling fluid-like regime exhibits dynamic fluid-like behavior.

[Figure 7. Power spectral density of the overall solid concentration at ECT upper plane for different flow regimes: (a) $u_{g}=1.27 \mathrm{~mm} / \mathrm{s}$ in the fixed bed regime, $(b) u_{g}=3.40 \mathrm{~mm} / \mathrm{s}$ in the solid-like homogeneous fluidization regime, $(c) u_{g}=5.31 \mathrm{~mm} / \mathrm{s}$ in the uniform non-bubbling fluid-like fluidization regime, and $(d) u_{g}=7.85 \mathrm{~mm} / \mathrm{s}$ in the bubbling fluidization regime. The fluidized powder is FCC I.]

One may identify the flow regime by fingerprint of either the dominant frequency or the typical magnitude of the power spectral density or both. ${ }^{49}$ Neglecting the dominant frequency, it is noted from Figure 7 that the order of the magnitude of the power spectral density increases greatly from $1 \times 10^{-4}$ in the fixed bed to 0.01 in the solid-like homogeneous fluidization regime and then to 0.1 in the uniform non-bubbling fluid-like fluidization regime and finally to 10 in the bubbling regime. This finding is in good agreement with the $\mathrm{Sd}$ of the overall solid concentration as shown in Figure 4.

[Figure 8. Power spectral density of the overall solid concentration at ECT upper plane when $u_{g}$ is about 3 times the $u_{m b}$. The fluidized powder is FCC I.] 


\subsection{Pressure fluctuation results}

Figure 9 shows the bed pressure drop normalized by particle weight per unit cross-sectional area $\left(\Delta p_{n}\right)$ as a function of $u_{g}$ in both a fluidization process (solid black line with black squares in Figure 9, and the repeated experimental results in three separate measurements are shown in Figure 5c) and a defluidization process (solid red line with red triangles in Figure 9) for FCC I powder. Here, the bed pressure drop is calculated as the time averaged pressure fluctuation signal measured at a certain velocity. Note that the regime regions shown in Figure 9 are divided following the aforementioned visual observations and ECT experimental results, and so are the transition velocities. It can be seen that in the fluidization process, the bed pressure drop first increases with increasing $u_{g}$, and when $u_{g}$ reaches $u_{m f}$, the pressure drop attains a maximum with the value larger than 1 (about 1.08), that is to say, a pressure overshoot over the particle weight per unit crosssectional area is seen. It has been found that both friction and inter-particle cohesive forces are responsible for this overshoot, ${ }^{21,36,50,51}$ and a qualitative understanding is that the stronger the friction or inter-particle cohesive forces, the higher the overshoot. ${ }^{36}$ Therefore, the maximum normalized pressure drop here, 1.08 , is closely related to the magnitude of the friction and interparticle cohesive forces. Upon further increasing $u_{g}$, the pressure drop finally decreases to level off at a value very close to 1 , indicating the bed is fluidized. While in the defluidization process, the normalized pressure drop first keeps constant in the bubbling and uniform non-bubbling fluidlike fluidization regimes. Upon decreasing $u_{g}$, the pressure drop decreases gradually in the solidlike homogeneous fluidization state, and further decreases sharply in the fixed bed regime. The 
different evolution of the bed pressure drop profile between the fluidization and defluidization processes is attributed to the hysteresis effect. ${ }^{7,19,50-53}$ As can be seen from Figure 9, the hysteresis is only pronounced in the fixed bed and solid-like homogeneous fluidization regimes. While in the uniform non-bubbling fluid-like and bubbling fluidization regimes, the pressure drop profiles in the fluidization and defluidization processes almost coincide with each other. This suggests the existence of permanent particle-particle contacts or yield stresses ${ }^{7}$ in both the fixed bed and solidlike homogeneous fluidization regimes. When $u_{g}$ is further increased to the uniform non-bubbling fluid-like and bubbling fluidization regimes, the yield stresses vanish along with the sustained particle-particle collisions. ${ }^{14,16}$ In this way, particles are free floating in the bed, leading to the flow resistance the same as particles settling in a defluidization process. From Figure 9, it is also clear that the $u_{m f}$ identified from the bed pressure drop profiles, either at the point where the pressure drop across the bed becomes sufficient to balance the particle weight in a fluidization process ${ }^{27,33,36}$ or at the intersection point of two curves (two dashed cyan lines in Figure 9) fitted for the fixed bed and bubbling bed in a defluidization process, ${ }^{48,54}$ is the same as that measured by ECT (see Figures 4$){ }^{41}$

[Figure 9. Normalized pressure drop and $S d$ of the pressure drop against $u_{g}$. The fluidized powder is FCC I.]

Similar to Figure 3, Figure 10 shows the transient bed pressure drop signals for different flow regimes. The ordinate range of Figures 10a, 10b, and 10c is also set to be the same, say $0.03 \mathrm{kPa}$. 
It can be seen that the bed pressure drop in the fixed bed regime fluctuates around a mean value less than the particle weight per unit cross-sectional area (about $3.09 \mathrm{~N} / \mathrm{m}^{2}$ ), indicating that the particle weight is partly supported by frictional stresses. While in other three regimes, the bed pressure drop fluctuates around a value slightly less than the particle weight per unit crosssectional area (also see Figure 9). Such an offset in pressure drop was also found in previous numerical and experimental studies, ${ }^{19,55}$ implying that a small part of particles is supported by the distributor and wall. However, unlike the overall solid concentration signals as shown in Figure 3, it is difficult to distinguish among the fixed bed, solid-like homogeneous fluidization, and uniform non-bubbling fluid-like fluidization regimes from the fluctuation of the bed pressure drop signals, because the amplitude and frequency of the fluctuation are similar in these three regimes. Only when the bed is in the bubbling regime, a large fluctuation begins to prevail.

[Figure 10. Sampled signals of the bed pressure drop for different flow regimes: (a) $u_{g}=1.91$ $\mathrm{mm} / \mathrm{s}$ in the fixed bed regime, $(b) u_{g}=3.40 \mathrm{~mm} / \mathrm{s}$ in the solid-like homogeneous fluidization regime, (c) $u_{g}=5.31 \mathrm{~mm} / \mathrm{s}$ in the uniform non-bubbling fluid-like fluidization regime, and (d) $u_{g}$ $=7.85 \mathrm{~mm} / \mathrm{s}$ in the bubbling fluidization regime. The fluidized powder is FCC I.]

To further test the fluctuation of the bed pressure drop signals, Figure 9 shows the $\mathrm{Sd}$ of the bed pressure drop during a fluidization process for FCC I powder (solid blue line with blue dots in Figure 9, and the repeated experimental results in three separate measurements are shown in Figure 5d). Besides, Sd of the pressure drop across the distributor itself (i.e., in an empty bed) as a function 
of $u_{g}$ is also shown as a baseline (solid black line with black diamonds in Figure 9) to evaluate the noise level of the pressure drop signals. Clearly, the Sd of the pressure drop in the empty bed shows weak dependence on $u_{g}$, suggesting that the $\mathrm{Sd}$ in the fluidization process can represent the dynamic behavior in the bed. It can be seen that the Sd of the pressure drop across the bed keeps small in the fixed bed, solid-like homogeneous fluidization, and uniform non-bubbling fluid-like fluidization regimes, while large fluctuations are observed in the bubbling regime, which is in line with the results shown in Figure 10. At the regime transition points, it is noted that the transition from the fixed bed regime to the solid-like homogeneous fluidization state is very smooth, while a local maximum is seen on the transition between the solid-like homogeneous and uniform nonbubbling fluid-like fluidization regimes. Another interesting finding is that there is a peak in the profile of the Sd in the fixed bed region, which is even higher than that on the transition between the solid-like homogeneous and uniform non-bubbling fluid-like fluidization regimes. The peak is suspected to be associated with the break-up of chain-like mechanical structures formed in the fixed bed, ${ }^{11,19,21,50}$ however, detailed mechanisms are not known at present, which anyway deserve further research.

[Figure 11. Power spectral density of the bed pressure drop for different flow regimes: $(a) u_{g}=$ $1.27 \mathrm{~mm} / \mathrm{s}$ in the fixed bed regime, $(b) u_{g}=3.40 \mathrm{~mm} / \mathrm{s}$ in the solid-like homogeneous fluidization regime, (c) $u_{g}=5.31 \mathrm{~mm} / \mathrm{s}$ in the uniform non-bubbling fluid-like fluidization regime, and (d) $u_{g}$ $=7.85 \mathrm{~mm} / \mathrm{s}$ in the bubbling fluidization regime. The fluidized powder is FCC I.] 
Figure 11 shows the power spectral density of the bed pressure drop for different flow regimes. Consistent with the Sd information as shown in Figure 9, except that the typical magnitude in the bubbling regime is much larger, there is no big difference among the fixed bed, solid-like homogeneous fluidization, and uniform non-bubbling fluid-like fluidization regimes in terms of either the dominant frequency or the magnitude of the power spectral density. Nevertheless, it is worthwhile mentioning that the dominant frequency of the pressure drop in the bubbling regime is similar to that obtained from the overall solid concentration by ECT (see Figure 7).

\subsection{Comparison among the used three measurement techniques}

Visual observation from the camera, ECT, and pressure fluctuation are three widely used techniques developed for the measurement of hydrodynamic behavior of gas-solid fluidized beds. ${ }^{56}$ In this section, a short comparison among these three techniques, with emphasis on their ability and performance to identify different flow regimes involved in this research, is presented.

Visualization is the most intuitive and straightforward way to study the gas-solid flow. A further detailed investigation is always conducted on the basis of visualization. For example, the popular Geldart's particle classification was established based on the visual observations of different fluidization behaviors characterized by different types of particles, ${ }^{1}$ and then many numerical, theoretical, and experimental works were performed to explore the mechanisms underlying this classification. Similarly, the investigation in this research was stimulated and triggered by the successful observation of the existence of both solid-like and fluid-like states in the homogeneous fluidization regime of Geldart A particles when a very small adjustment in $u_{g}$ was used in experiments. The identification of different flow regimes was also first based on visual 
observations. However, the application of visualization method is only restricted to systems for visual access. Therefore, it is necessary to see whether other technologies that can work at visual inaccessible environments, such as ECT and pressure fluctuation measurements, can identify solidlike and fluid-like states in the homogeneous fluidization regime.

Both time and frequency domain analysis were performed on the overall solid concentration signals measured by ECT and pressure drop signals obtained from pressure fluctuation measurements. It was found that the ECT experimental results are consistent with those obtained from visual observations. More specifically, two indicators, i.e., the $\mathrm{Sd}$ and the typical magnitude of the power spectral density of the overall solid concentration, provide a robust and reliable way to identify different flow regimes. However, no significant changes are found in both time and frequency domain analysis of the bed pressure drop signals among the fixed bed, solid-like homogeneous fluidization, and uniform non-bubbling fluid-like fluidization regimes, and only at a velocity that is a little higher than the critical velocity $u_{c}$, a local maximum is seen in the curve of the $\mathrm{Sd}$ of the bed pressure drop against $u_{g}$.

The different performance and ability between ECT and pressure fluctuation measurements lie in their different measurement strategies. In ECT, the gas-solid flow information on a cross section is interrogated by an array of electrodes from different view angles, while the pressure fluctuation measurement is only from a single point near the wall. Therefore, the ECT results consist of more information regarding the hydrodynamic behavior of fluidized beds. From this point of view, it is not surprising that it is ECT, but not pressure fluctuation method, that is sensitive to the small changes among the fixed bed, solid-like homogeneous fluidization, and uniform non- 
bubbling fluid-like fluidization regimes. Ye et al. ${ }^{36}$ also proved in their DPM simulations that the temporal fluctuation of pressure drop is not a reliable indicator to characterize the transition from the homogeneous fluidization to bubbling fluidization. Even so, ignoring the fluctuation, the hysteresis effect of the bed pressure drop between increasing and decreasing $u_{g}$, as a welldocumented phenomenon for gas-solid fluidized beds, can provide additional valuable insights on the homogeneous fluidization: the yield stresses exist in the solid-like homogeneous fluidization state, while they vanish in the uniform non-bubbling fluid-like fluidization regime. However, such a knowledge can never be deduced from ECT experimental results. There are many excellent comparative studies between both methods for gas-solid fluidized beds measurement in literature. ${ }^{33,48}$ All these studies, including this research, suggest that even though ECT provides more sensitive results and more information compared to the pressure fluctuation method, it is still good to combine both techniques in practical applications.

\subsection{Fluidization behavior differences between Geldart A and modified Geldart C particles}

It has been anticipated that the plot of the $\mathrm{Sd}$ of the overall solid concentration measured by ECT against $u_{g}$ can be used to identify the fixed bed, solid-like homogeneous fluidization, uniform non-bubbling fluid-like fluidization, and bubbling fluidization regimes. Figure 12 shows time domain analysis of the overall solid concentration for $\mathrm{FCC}$ II and $\mathrm{Al}_{2} \mathrm{O}_{3}$ powders. As can be seen, the profiles of both the average and Sd of the overall solid concentration are similar to those shown in Figure 4 for FCC I powder. Especially, the Sd of the overall solid concentration also shows three plateaus. Note that visual observations and pressure fluctuation measurements were also performed on these two powders, and the results are similar to FCC I powder. The transition 
velocities, i.e., $u_{m f}, u_{c}$, and $u_{m b}$, as identified from the terminal point of each plateau in the curve of the $\mathrm{Sd}$ of the overall concentration, are listed alongside those calculated from the empirical correlation of Abrahamsen and Geldart ${ }^{17}$ in Table 2. Clearly, a good agreement with the maximum relative error less than $2 \%$ for both $u_{m f}$ and $u_{m b}$ is shown. Therefore, it is evident that the existence of both solid-like and fluid-like states is not a particular case for FCC I powder only, but a general rule for true Geldart A particles. That is, the existence of solid-like and fluid-like states in the homogeneous fluidization regime is now experimentally validated for Geldart A particles.

[Figure 12. Time domain analysis of the overall solid concentration at ECT upper plane for (a) FCC II powder and (b) $\mathrm{Al}_{2} \mathrm{O}_{3}$ powder.]

[Table 2. Transition velocities obtained from experiments and the empirical correlation of Abrahamsen and Geldart for different powders]

Comparing the experimental results of the studied three Geldart A particles to reports on the nanoparticles-modified Geldart C particles, ${ }^{14,16}$ it can be concluded that fluidization behaviors of both in the solid-like homogeneous fluidization and uniform non-bubbling fluid-like fluidization regimes are similar, say the bed remains static in the solid-like state while some void and cavity structures appear in the fluid-like state. However, there are also some apparent differences. For example, a $40 \%$ expansion of the initial height can be achieved for the modified Geldart $\mathrm{C}$ particles, while only about $25 \%$ for the studied Geldart A particles. Also, the maximum free volume is 0.85 
for the modified Geldart C particles, but only $0.45 \sim 0.48$ for the studied Geldart A particles. Regarding the ratio of $u_{m b}$ to $u_{m}$, values of $2.7,3$, and 1.7 , which are much lower than that of the modified Geldart C particles, say about 40, are dominant for the studied FCC I, FCC II, and $\mathrm{Al}_{2} \mathrm{O}_{3}$ powders, respectively.

While there has been a consensus that a dynamic aggregation process enables stable fluidization for the nanoparticles-modified Geldart C particles, ${ }^{16,20-23}$ it is still an open question whether Geldart A particles aggregate in the homogeneous expansion regime. ${ }^{24-26}$ Despite the ambiguity, a sedimentation model originally developed by Valverde's research group for the modified Geldart $\mathrm{C}$ particles ${ }^{22,23}$ was used to try to estimate the possible aggregate size of the studied Geldart A particles in the homogeneous fluidization regime. First, the particles were driven into the bubbling regime for a sufficiently long time. Next, $u_{g}$ was set down to a certain value below the $u_{m b}$. Once the particles were in a steady state for a given $u_{g}$, the gas flow was abruptly shut off and the particles settled down. During the sedimentation process, the bed height was continuously recorded using the camera at a frequency of $25 \mathrm{~Hz}$. It was found that the settling velocity keeps constant in the initial period, and then slows down due to stresses carried by interparticle forces. The initial settling velocity $\left(u_{s}\right)$ for different $u_{g}$ was measured. Finally, the relationship between $u_{s}$ and the overall solid concentration $\beta$ before the sedimentation process can be expressed by Equation 12, a modified Richardson-Zaki law with the consideration of aggregation in gas-solid fluidization, ${ }^{22}$

$$
\frac{u_{s}}{u_{t}}=\frac{N_{p}}{r}\left(1-\frac{r^{3}}{N_{p}} \beta\right)^{5.6}
$$

where $N_{p}$ is the number of particles aggregated in an aggregate, $r$ is the ratio of the average 
aggregate size to the particle size, and $u_{t}$ is the terminal velocity of a single particle, which can be calculated from the well-known Stokes equation in the laminar regime: ${ }^{22}$

$$
u_{t}=\frac{\left(\rho_{p}-\rho_{g}\right) g d_{p}^{2}}{18 \mu}
$$

Fitting the measured $u_{s}$ and $\beta$ to Equation 12 yields $N_{p}$ and $r$, which can be further used to predict the average aggregate size $\left(d_{a g g}\right)$ and the packing fraction of particles in the aggregate $\left(\beta_{a g g}\right)$, respectively, by Equations 14 and 15:22

$$
\begin{gathered}
d_{a g g}=r d_{p} \\
\beta_{a g g}=\frac{N_{p}}{r^{3}}
\end{gathered}
$$

The model, corroborated by direct visualization of samples taken from beds, gave $N_{p} \approx 100$, $d_{a g g} \approx 100 \mu \mathrm{m}$, and $\beta_{a g g} \approx 0.51$ for the $8.53-\mu \mathrm{m}$ xerographic toner, ${ }^{16,22,23}$ that is to say, $100-\mu \mathrm{m}$ particle aggregates, with each formed by about 100 raw toner particles, were fluidized in the bed.

Figure 13a shows the sedimentation experimental and fitted results for FCC I powder. As can be seen, Equation 12 fits the experimental data well, giving for the best fit $N_{p}=1.35$ and $r=1.08$. The average aggregate size $d_{a g g}$ is then predicted by Equation 14 to be about $74.9 \mu \mathrm{m}$, which is very close to the average particle diameter, $69.5 \mu \mathrm{m}$. The packing fraction of particles in the aggregate $\beta_{a g g}$ is calculated by Equation 15 to be about 1.07 . Both $d_{a g g}$ and $\beta_{a g g}$ indicate that, different from the modified Geldart C particles, the studied Geldart A particles do not display aggregation structures in the homogeneous fluidization regime.

[Figure 13. Sedimentation experimental and fitted results using (a) Equation 12 and (b) Equation 16 for FCC I powder.] 
In addition to the modified Richardson-Zaki law, the sedimentation experimental data are also fitted to the original Richardson-Zaki law: ${ }^{13}$

$$
\frac{u_{s}}{u_{t}{ }^{*}}=(1-\beta)^{n^{*}}
$$

where $u_{t}{ }^{*}$ is the fitted terminal velocity and $n^{*}$ is an empirical parameter. The results are shown in Figure 13b. The fitted $u_{t}{ }^{*}$ and $n^{*}$ are $0.18 \mathrm{~m} / \mathrm{s}$ and 4.72 , respectively. It is noted that the fitted $u_{t}{ }^{*}$ is identical to the one obtained from the Stokes equation, and the fitted $n^{*}$ is comparable to the one obtained from liquid fluidized beds, $4.6 .{ }^{13}$ Barreto et al. ${ }^{57}$ fluidized five different Geldart A particles and also found that the values of $n^{*}$ were close to those obtained from empirical correlations developed for liquid fluidized beds. As the original Richardson-Zaki law itself was obtained on the assumption of homogeneous distribution of individual particles, the results extracted from Figure 13b confirm again that no particle aggregates present for the studied Geldart A particles. The sedimentation experimental and fitted results using Equations 12 and 16 performed on FCC II $\left(N_{p}=1.33, r=1.07, u_{t}=0.16 \mathrm{~m} / \mathrm{s}, u_{t}{ }^{*}=0.17 \mathrm{~m} / \mathrm{s}\right.$, and $\left.n^{*}=4.76\right)$ and $\mathrm{Al}_{2} \mathrm{O}_{3}$ $\left(N_{p}=1.49, r=1.12, u_{t}=0.29 \mathrm{~m} / \mathrm{s}, u_{t}{ }^{*}=0.26 \mathrm{~m} / \mathrm{s}\right.$, and $\left.n^{*}=4.64\right)$ powders also show a similar aggregation behavior to FCC I powder.

There is no doubt that the formation of particle agglomerates for the modified Geldart $\mathrm{C}$ particles is caused by the existence of significant inter-particle forces, the magnitude of which, however, is very difficult to measure or predict. Valverde and Castellanos ${ }^{23}$ estimated the interparticle forces of the nanoparticles-modified Geldart $\mathrm{C}$ particles to be about $2 \mathrm{nN}$. The Bond number $(B o)$, defined as the ratio of inter-particle forces to particle weight, of the $8.53-\mu \mathrm{m}$ 
xerographic toner can be then calculated to be as high as 550. For the studied Geldart A particles, a modified Harrison equation is introduced here to estimate $B o:{ }^{23}$

$$
\frac{D_{b m}}{d_{a g g}}=\frac{\rho_{p}^{2} g d_{p}^{3}}{18^{2} 0.7^{2} \mu^{2}} B o^{\left(2 \frac{\ln N_{p}}{\ln r}-3\right) /\left(\frac{\ln N_{p}}{\ln r}+2\right)}
$$

where $D_{b m}$ is the maximum microscopic bubble size in the uniform non-bubbling fluid-like fluidization regime. It has been proved that the studied Geldart A particles do not aggregate. Therefore, $d_{a g g}$ is replaced by $d_{p}$ in Equation 17 . Geldart ${ }^{1}$ suggested that an obvious bubble indicating the onset of the bubbling regime has a diameter of at least $5 \mathrm{~mm}$ in a bed with the size the same as that used in this work. Figure $2 \mathrm{c}$ and Video S2a also demonstrate that the diameter of the microscopic bubbles in the uniform non-bubbling fluid-like fluidization regime is smaller than $5 \mathrm{~mm}$. In these regards, $D_{b m}$ takes the value of $5 \mathrm{~mm}$. With the known $D_{b m}$ and $d_{a g g}, B o$ can be then back-calculated from Equation 17. In this way, Bo of FCC I, FCC II, and $\mathrm{Al}_{2} \mathrm{O}_{3}$ powders is estimated to be about $0.66,0.90$, and 0.18 , respectively, the magnitude of which is in accordance with the postulate that for Geldart A particles, the inter-particle forces and particle weight are of comparable magnitude. ${ }^{58}$ Clearly, Bo of the studied Geldart A particles is much lower than that of the modified Geldart $\mathrm{C}$ particles (this is right if $\mathrm{Bo}$ of the modified Geldart $\mathrm{C}$ particles is also backcalculated from Equation 17: assuming that $D_{b m} / d_{a g g}$ is in the range 1 10, which is typical for the modified Geldart C particles, ${ }^{23}$ Bo of the 8.53- $\mu \mathrm{m}$ xerographic toner is in the range 60 7000, which is also much larger than that of the studied Geldart A particles). It can be then concluded that the relative importance of inter-particle forces is responsible for the different apparent fluidization characteristics and aggregation behaviors between these two kinds of particles. Furthermore, referring back to Table 2, it is noted that the interval length of the solid like homogeneous 
fluidization and uniform non-bubbling fluid-like fluidization regimes is different among the three studied Geldart A particles. Especially, the interval of the solid-like homogeneous fluidization regime for $\mathrm{Al}_{2} \mathrm{O}_{3}$ powder is much shorter than that for FCC I and FCC II powders. Relating this finding to the values of $B o$ suggests that the extension of both the solid-like and fluid-like states is also influenced by $B o$. However, a quantitative description on the effect of $B o$ is not possible at present.

The ratio $D_{b m} / d_{a g g}$ has been used as a criterion by Valverde and Castellanos ${ }^{23}$ to predict the types of gas fluidization of cohesive particles. It has been claimed that only when $D_{b m} / d_{a g g}<10$, can the uniform non-bubbling fluid-like fluidization regime exist, otherwise the solid-like homogeneous fluidization regime will transit directly to the bubbling regime. However, using the values of $D_{b m}=5 \mathrm{~mm}$ and $d_{a g g}=d_{p}, D_{b m} / d_{a g g}$ of the studied three Geldart A particles is in the range

70 75, which already exceeds the upper bound, 10. But indeed, the uniform non-bubbling fluidlike fluidization regime is experimentally observed in this work. It implies that the criterion, originally developed for the modified Geldart $\mathrm{C}$ particles, may not suit for Geldart A particles, and thus a new criterion that can be applied to both the modified Geldart $\mathrm{C}$ and true Geldart A particles is needed.

\section{Conclusions}

In this work, camera recording, ECT, and pressure fluctuation were used to experimentally investigate the homogeneous fluidization characteristics of three typical Geldart A powders. Both time and frequency domain analysis were performed on the overall solid concentration signals 
measured by ECT and bed pressure drop signals obtained from pressure fluctuation measurements. The ability and performance of the used three measurement techniques to identify different flow regimes were compared. Based on this work, the following conclusions are drawn:

(1) For the first time, the existence of both solid-like and fluid-like states in the homogeneous expansion regime was experimentally verified for Geldart A particles. In both states, the bed expands gradually with a stable bed height. However, there are also differences between them: in the solid-like state, the bed remains static just like that in the fixed bed regime, while some void and cavity structures appear in the fluid-like state.

(2) The Sd and power spectral density of the overall solid concentration are two reliable indicators to identify different flow regimes. More specifically, the plot of the Sd of the overall solid concentration against $u_{g}$ shows three plateaus, corresponding to the fixed bed, solid-like homogeneous fluidization, and uniform non-bubbling fluid-like fluidization regimes. The gas velocity at the terminal point of each plateau is $u_{m f}, u_{c}$, and $u_{m b}$, and the obtained transition velocities show good agreement with the empirical correlation. As for the power spectral density, it increases with orders of magnitude as the regime evolution.

(3) The hysteresis effect of the bed pressure drop indicates the existence of permanent yield stresses in both the fixed bed and solid-like homogeneous fluidization regimes, while the yield stresses vanish along with the sustained particle-particle collisions in the uniform non-bubbling fluid-like fluidization and bubbling fluidization regimes.

(4) Time and frequency domain analysis of the bed pressure drop fluctuation signals are not as reliable as ECT to identify different flow regimes involved in this research. 
(5) The different performance of ECT and pressure fluctuation methods lies in their different measurement strategies. It is good to combine both techniques to investigate gas-solid flow characteristics in practical applications.

(6) Once the existence of both states is verified, it is reasonable to speculate that the mechanisms of the homogeneous fluidization of Geldart A particles have two distinct factors: one dominated by inter-particle forces in the solid-like state and the other by fluid dynamics in the fluid-like state.

(7) The sedimentation experiments indicate that different from the modified Geldart C particles, no particle aggregates present in the homogeneous fluidization regime for Geldart A particles. The relative importance of inter-particle forces is responsible for the different apparent fluidization characteristics and aggregation behaviors between these two kinds of particles.

As the aim of this paper is to experimentally verify the existence of solid-like and fluid-like states in the homogeneous fluidization regime of Geldart A particles using different measurement techniques, the work presented is intended to be the first step in a systematic study. Further researches are still needed to (1) understand the flow hydrodynamics behind these two states, (2) explore the effect of $B o$ on the extension of both states, and (3) develop a new criterion that can be applied to both the modified Geldart C and true Geldart A particles. These works are currently underway.

\section{NOMENCLATURE}

\section{Roman letters}




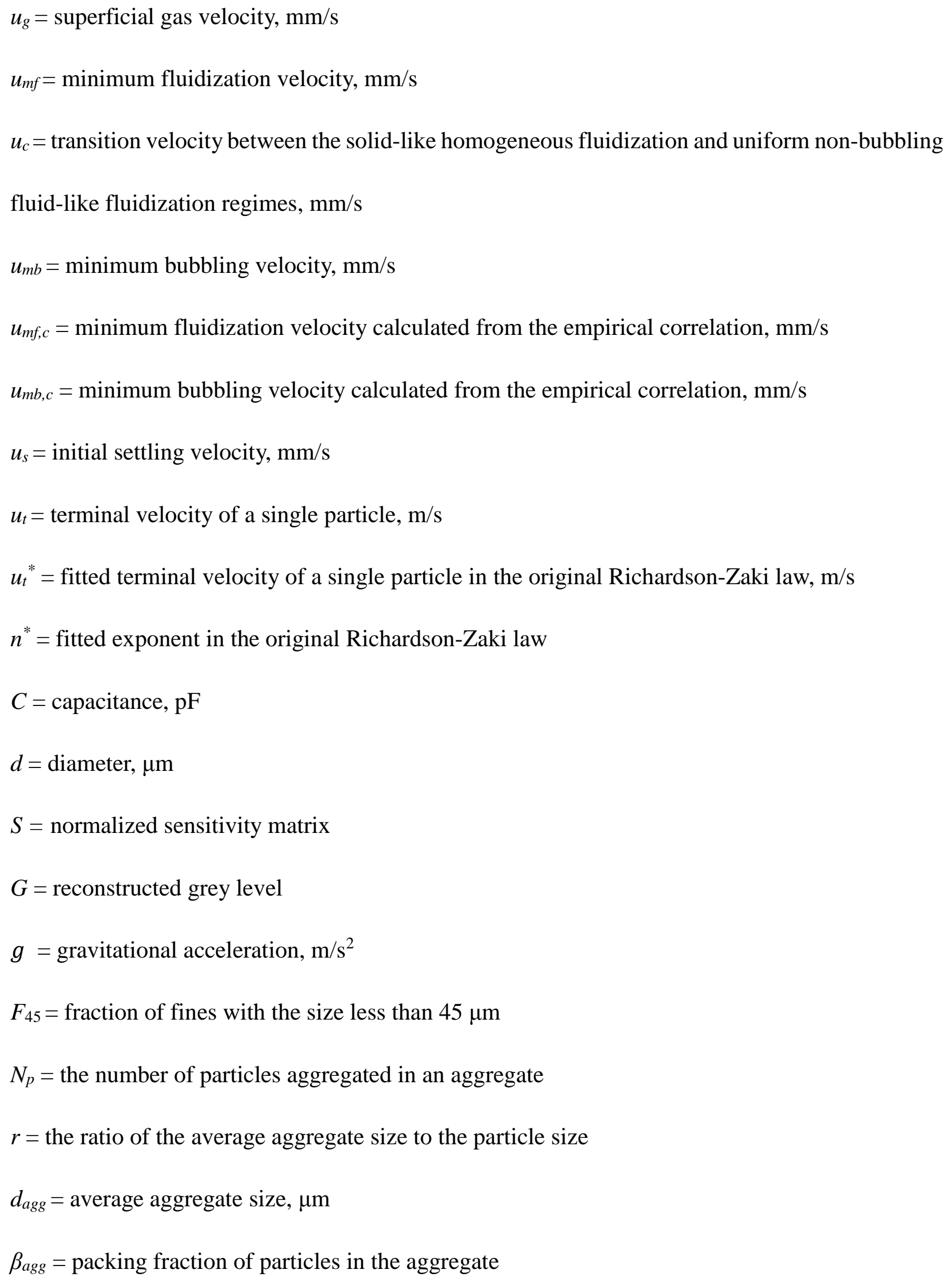


$D_{b m}=$ maximum microscopic bubble size in the non-bubbling fluid-like regime, $\mathrm{mm}$

$B o=$ Bond number

Greek letters

$\alpha=$ step length in the projected Landweber iteration algorithm

$\lambda=$ normalized capacitance

$u_{\lambda}=$ identity vector (a vector of ones)

$\beta=$ overall solid concentration

$\theta=$ packed bed solid concentration

$\rho=$ density, $\mathrm{kg} / \mathrm{m}^{3}$

$\mu=$ viscosity, $\mathrm{Pa} \cdot \mathrm{s}$

\section{Subscripts}

$g=$ air

$p=$ particles

$L=$ low calibration

$H=$ high calibration

$M=$ measurement

\section{Abbreviations}

$\mathrm{ECT}=$ electrical capacitance tomography

$\mathrm{FCC}=$ fluid catalytic cracking

MTO $=$ methanol to olefins

$\mathrm{LBP}=$ linear back projection 
$\mathrm{DPM}=$ discrete particle model

$\mathrm{Sd}=$ standard deviation

$\mathrm{NSR}=$ noise-to-signal ratio, $1 / \mathrm{dB}$

$\mathrm{SNR}=$ signal-to-noise ratio, $\mathrm{dB}$

$\mathrm{FFT}=$ fast Fourier transformation

\section{Acknowledgments}

This work is part of a research program financially supported by the Newton Advanced Fellowship of the Royal Society, UK under Grant No. NA140308 and the National Natural Science Foundation of China under Grant No. 91334205.

\section{Supporting Information}

Videos taken in different flow regimes by the camera are shown in Supporting Information (AVI).

Video $\mathrm{S} 1 \mathrm{a}$ is in the fixed bed regime, Video $\mathrm{S} 1 \mathrm{~b}$ is in the solid-like homogeneous fluidization regime, Video S2a is in the uniform non-bubbling fluid-like fluidization regime, and Video S2b is in the bubbling fluidization regime.

\section{References}

(1) Geldart, D., Types of gas fluidization. Powder Technol. 1973, 7, 285.

(2) Yang, W.-C., Handbook of fluidization and fluid-particle systems; Marcel Dekker Inc.: New 
York, 2003.

(3) Sundaresan, S., Instabilities in fluidized beds. Annu. Rev. Fluid Mech. 2003, 35, 63.

(4) Foscolo, P. U.; Gibilaro, L. G., A fully predictive criterion for the transition between particulate and aggregate fluidization. Chem. Eng. Sci. 1984, 39, 1667.

(5) Rietema, K., The effect of interparticle forces on the expansion of a homogeneous gas-fluidised bed. Chem. Eng. Sci. 1973, 28, 1493.

(6) Garg, S. K.; Pritchett, J. W., Dynamics of gas- fluidized beds. J. Appl. Phys. 1975, 46, 4493.

(7) Tsinontides, S. C.; Jackson, R., The mechanics of gas fluidized beds with an interval of stable fluidization. J. Fluid Mech. 1993, 255, 237.

(8) Menon, N.; Durian, D. J., Particle motions in a gas-fluidized bed of sand. Phys. Rev. Lett. 1997, $79,3407$.

(9) Cody, G. D.; Goldfarb, D. J.; Storch, G. V.; Norris, A. N., Particle granular temperature in gas fluidized beds. Powder Technol. 1996, 87, 211.

(10) Fortes, A. F.; Caldas, P.; Gallo, J. V., Particle aggregation and the van der Waals forces in gassolids fluidization. Powder Technol. 1998, 98, 201.

(11) Ye, M.; van der Hoef, M. A.; Kuipers, J. A. M., A numerical study of fluidization behavior of Geldart A particles using a discrete particle model. Powder Technol. 2004, 139, 129.

(12) Ye, M.; van der Hoef, M. A.; Kuipers, J. A. M., Discrete particle simulation of the homogeneous fluidization of Geldart A particles, In Proceedings of Fluidization XI, Naples, Italy, 2004, 699.

(13) Oke, O.; Lettieri, P.; Mazzei, L., An investigation on the mechanics of homogeneous 
expansion in gas-fluidized beds. Chem. Eng. Sci. 2015, 127, 95.

(14) Valverde, J. M.; Castellanos, A.; Quintanilla, M. A. S., Self-diffusion in a gas-fluidized bed of fine powder. Phys. Rev. Lett. 2001, 86, 3020.

(15) Valverde, J. M.; Castellanos, A.; Mills, P.; Quintanilla, M. A. S., Effect of particle size and interparticle force on the fluidization behavior of gas-fluidized beds. Phys. Rev. E 2003, 67, 051305.

(16) Valverde, J. M.; Quintanilla, M. A. S.; Castellanos, A.; Mills, P., Experimental study on the dynamics of gas-fluidized beds. Phys. Rev. E 2003, 67, 016303.

(17) Abrahamsen, A. R.; Geldart, D., Behaviour of gas-fluidized beds of fine powders part I. Homogeneous expansion. Powder Technol. 1980, 26, 35.

(18) Ye, M., Multi-level modeling of dense gas-solid two-phase flows; University of Twente: Enschede, 2005.

(19) Espin, M. J.; Quintanilla, M. A. S.; Valverde, J. M., Magnetic stabilization of fluidized beds: Effect of magnetic field orientation. Chem. Eng. J. 2017, 313, 1335.

(20) Valverde, J. M.; Castellanos, A., Effect of vibration on agglomerate particulate fluidization. AlChE J. 2006, 52, 1705.

(21) Valverde, J. M., Fluidization of fine powders: Cohesive versus dynamical aggregation; Springer: Dordrecht Heidelberg New York London, 2013.

(22) Valverde, J. M.; Quintanilla, M. A. S.; Castellanos, A.; Mills, P., The settling of fine cohesive powders. Europhys. Lett. 2001, 54, 329.

(23) Valverde, J. M.; Castellanos, A., Types of gas fluidization of cohesive granular materials. 
Phys. Rev. E 2007, 75, 031306.

(24) Ye, M.; Wang, J. W.; van der Hoef, M. A.; Kuipers, J. A. M., Two-fluid modeling of Geldart A particles in gas-fluidized beds. Particuology 2008, 6, 540.

(25) Wang, J. W.; van der Hoef, M. A.; Kuipers, J. A. M., Why the two-fluid model fails to predict the bed expansion characteristics of Geldart A particles in gas-fluidized beds: A tentative answer. Chem. Eng. Sci. 2009, 64, 622.

(26) Sande, P. C.; Ray, S., Fine mesh Computational Fluid Dynamics study on gas-fluidization of Geldart A particles: Homogeneous to bubbling bed. Ind. Eng. Chem. Res. 2016, 55, 2623.

(27) Xie, H.-Y.; Geldart, D., Fluidization of FCC powders in the bubble-free regime: effect of types of gases and temperature. Powder Technol. 1995, 82, 269.

(28) Yang, W. Q.; York, T. A., New AC-based capacitance tomography system. IEE Proc-Sci. Meas. Technol. 1999, 146, 47.

(29) Yang, W. Q.; Peng, L. H., Image reconstruction algorithms for electrical capacitance tomography. Meas. Sci. Technol. 2003, 14, R1.

(30) Guo, Q.; Meng, S. H.; Wang, D. H.; Zhao, Y. F.; Ye, M.; Yang, W. Q.; Liu, Z. M., Investigation of gas-solid bubbling fluidized beds using ECT with a modified Tikhonov regularization technique. AlChE J. 2018, 64, 29.

(31) Yang, W. Q.; Spink, D. M.; York, T. A.; McCann, H., An image-reconstruction algorithm based on Landweber's iteration method for electrical-capacitance tomography. Meas. Sci. Technol. 1999, 10, 1065.

(32) Wang, M., Industrial tomography: systems and applications; Elsevier: 2015. 
(33) Zhang, J. L.; Mao, M. X.; Ye, J. M.; Wang, H. G.; Yang, W. Q., Investigation of wetting and drying process in a gas-solid fluidized bed by electrical capacitance tomography and pressure measurement. Powder Technol. 2016, 301, 1148.

(34) Azzopardi, B. J.; Abdulkareem, L. A.; Zhao, D.; Thiele, S.; da Silva, M. J.; Beyer, M.; Hunt, A., Comparison between Electrical Capacitance Tomography and Wire Mesh Sensor output for air/silicone oil flow in a vertical pipe. Ind. Eng. Chem. Res. 2010, 49, 8805.

(35) Azzopardi, B. J., Flow patterns: Does gas/solids flow pattern correspond to churn flow in gas/liquid flow. Ind. Eng. Chem. Res. 2008, 47, 7934.

(36) Ye, M.; van der Hoef, M. A.; Kuipers, J. A. M., The effects of particle and gas properties on the fluidization of Geldart A particles. Chem. Eng. Sci. 2005, 60, 4567.

(37) Ostrowski, K. L.; Luke, S. P.; Bennett, M. A.; Williams, R. A., Application of capacitance electrical tomography for on-line and off-line analysis of flow pattern in horizontal pipeline of pneumatic conveyer. Chem. Eng. J. 2000, 77, 43.

(38) Makkawi, Y. T.; Wright, P. C., Fluidization regimes in a conventional fluidized bed characterized by means of electrical capacitance tomography. Chem. Eng. Sci. 2002, 57, 2411.

(39) Zhang, W. B.; Cheng, Y. P.; Wang, C.; Yang, W. Q.; Wang, C.-H., Investigation on hydrodynamics of triple-bed combined circulating fluidized bed using Electrostatic sensor and Electrical Capacitance Tomography. Ind. Eng. Chem. Res. 2013, 52, 11198.

(40) Ge, R. H.; Ye, J. M.; Wang, H. G.; Yang, W. Q., Measurement of particle concentration in a Wurster fluidized bed by electrical capacitance tomography sensors. AlChE J. 2014, 60, 4051.

(41) Luo, Q.; Zhang, Y. L.; Zhao, Y. F.; Ye, M.; Liu, Z. M., Measuring minimum fluidization 
velocity of Geldart A particles by use of electrical capacitance tomography. J. Cent. South. Univ. 2016, 47, 3916, (in Chinese).

(42) Wang, J. W.; van der Hoef, M. A.; Kuipers, J. A. M., CFD study of the minimum bubbling velocity of Geldart A particles in gas-fluidized beds. Chem. Eng. Sci. 2010, 65, 3772.

(43) Girimonte, R.; Formisani, B., The minimum bubbling velocity of fluidized beds operating at high temperature. Powder Technol. 2009, 189, 74.

(44) Karimipour, S.; Pugsley, T., A critical evaluation of literature correlations for predicting bubble size and velocity in gas-solid fluidized beds. Powder Technol. 2011, 205, 1.

(45) Liu, W. M.; Yang, S.; Li, H. Z.; Zhu, Q. S., A transfer coefficient-based structure parameters method for CFD simulation of bubbling fluidized beds. Powder Technol. 2016, 295, 122.

(46) Zhang, Y.; Liang, Y. C.; Wang, C.-H., Hazard of electrostatic generation in a pneumatic conveying system: electrostatic effects on the accuracy of electrical capacitance tomography measurements and generation of spark. Meas. Sci. Technol. 2008, 19, 015502.

(47) Lungu, M.; Zhou, Y. F.; Wang, J. D.; Yang, Y. R., A CFD study of a bi-disperse gas-solid fluidized bed: Effect of the EMMS sub grid drag correction. Powder Technol. 2015, 280, 154.

(48) Li, X. X.; Jaworski, A. J.; Mao, X. A., Comparative study of two non-intrusive measurement methods for bubbling gas-solids fluidized beds: electrical capacitance tomography and pressure fluctuations. Flow Meas. Instrum. 2017, DOI: 10.1016/j.flowmeasinst.2017.08.002.

(49) Rao, S. M.; Zhu, K.; Wang, C.-H.; Sundaresan, S., Electrical Capacitance Tomography measurements on the pneumatic conveying of solids. Ind. Eng. Chem. Res. 2001, 40, 4216.

(50) Castellanos, A., The relationship between attractive interparticle forces and bulk behaviour in 
dry and uncharged fine powders. Adv. Phys. 2005, 54, 263.

(51) Galvin, J. E.; Benyahia, S., The effect of cohesive forces on the fluidization of aeratable powders. AlChE J. 2014, 60, 473.

(52) Bernard, M.; Climent, E.; Wachs, A., Controlling the quality of two-way Euler/Lagrange numerical modeling of bubbling and spouted fluidized beds dynamics. Ind. Eng. Chem. Res. $\mathbf{2 0 1 7}, 56,368$.

(53) Zhu, C.; Yu, Q.; Dave, R. N.; Pfeffer, R., Gas fluidization characteristics of nanoparticle agglomerates. AlChE J. 2005, 51, 426.

(54) Wilkinson, D., Determination of minimum fluidization velocity by pressure fluctuation measurement. Can. J. Chem. Eng. 1995, 73, 562.

(55) Wang, J. W.; van der Hoef, M. A.; Kuipers, J. A. M., Coexistence of solidlike and fluidlike states in a deep gas-fluidized bed. Ind. Eng. Chem. Res. 2010, 49, 5279.

(56) Sun, J. Y.; Yan, Y., Non-intrusive measurement and hydrodynamics characterization of gassolid fluidized beds: a review. Meas. Sci. Technol. 2016, 27, 112001.

(57) Barreto, G. F.; Yates, J. G.; Rowe, P. N., The measurement of emulsion phase voidage in gas fluidized beds of fine powders. Chem. Eng. Sci. 1983, 38, 345.

(58) Liu, P. Y.; LaMarche, C. Q.; Kellogg, K. M.; Hrenya, C. M., Fine-particle defluidization: Interaction between cohesion, Young's modulus and static bed height. Chem. Eng. Sci. 2016, $145,266$. 


\section{List of Table Captions}

Table 1. Physical properties of the powders

Table 2. Transition velocities obtained from experiments and the empirical correlation of Abrahamsen and Geldart for different powders 
Table 1. Physical properties of the powders

\begin{tabular}{|c|c|c|c|c|}
\hline Powder & $\begin{array}{c}\text { Density } \\
\left(\rho_{p}, \mathrm{~kg} / \mathrm{m}^{3}\right)^{\mathrm{a}}\end{array}$ & $\begin{array}{c}\text { Mean Particle } \\
\text { Diameter }\left(d_{p}, \mu \mathrm{m}\right)^{\mathrm{b}}\end{array}$ & $\begin{array}{c}\text { Fine Particles } \\
\text { Content }\left(F_{45}, \%\right)^{\mathrm{c}}\end{array}$ & Origin \\
\hline FCC I & $1300( \pm 50)$ & $69.5( \pm 0.6)$ & $16.4( \pm 0.5)$ & Beijing, China \\
\hline FCC II & $1260( \pm 40)$ & $66.3( \pm 0.7)$ & $22.5( \pm 0.4)$ & Qingdao, China \\
\hline $\mathrm{Al}_{2} \mathrm{O}_{3}$ & $1950( \pm 25)$ & $71.2( \pm 0.8)$ & $9.5( \pm 0.6)$ & Dalian, China \\
\hline \multicolumn{5}{|c|}{$\begin{array}{l}{ }^{\mathrm{a}} \text { The density of particles } \rho_{p} \text { was measured by water displacement technique. } \\
{ }^{\mathrm{b}} \text { The mean particle diameter } d_{p} \text { was calculated as the Sauter mean diameter. } \\
{ }^{\mathrm{c}} \text { The fine particles content } F_{45} \text { is the fraction of fines with the size less than } 45 \mu \mathrm{m} \text {. } \\
\text { The uncertainty in this Table is the standard deviation of the results in three separate } \\
\text { measurements. }\end{array}$} \\
\hline
\end{tabular}


Table 2. Transition velocities obtained from experiments and the empirical correlation of Abrahamsen and Geldart for different powders

\begin{tabular}{cccccc}
\hline Powder & $u_{m f}, \mathrm{~mm} / \mathrm{s}$ & $u_{c}, \mathrm{~mm} / \mathrm{s}$ & $u_{m b}, \mathrm{~mm} / \mathrm{s}$ & $u_{m f, c}, \mathrm{~mm} / \mathrm{s}^{*}$ & $u_{m b, c}, \mathrm{~mm} / \mathrm{s}^{*}$ \\
\hline FCC I & 2.55 & 4.03 & 7.00 & 2.57 & 7.08 \\
FCC II & 2.33 & 4.24 & 7.00 & 2.30 & 7.07 \\
$\mathrm{Al}_{2} \mathrm{O}_{3}$ & 3.99 & 5.01 & 6.88 & 3.95 & 6.96 \\
$*$ & $u_{m f}$ and $u_{m b}$ with the subscript $c$ mean the values calculated from the empirical \\
correlation of Abrahamsen and Geldart.
\end{tabular}




\section{List of Figure Captions}

Figure 1. (a) Experimental setup. (b) ECT sensor structure. (c) Particle size distribution of the powders. (d) Situation of the powders in the Geldart diagram.

Figure 2. Visual snapshots taken by the camera: (a) $u_{g}=1.27 \mathrm{~mm} / \mathrm{s}$ in the fixed bed regime, (b) $u_{g}$ $=3.18 \mathrm{~mm} / \mathrm{s}$ in the solid-like homogeneous fluidization regime, (c) $u_{g}=5.09 \mathrm{~mm} / \mathrm{s}$ in the uniform non-bubbling fluid-like fluidization regime, and (d) $u_{g}=7.85 \mathrm{~mm} / \mathrm{s}$ in the bubbling fluidization regime. The fluidized powder is FCC I.

Figure 3. Sampled signals of the overall solid concentration at ECT upper plane for different flow regimes: (a) $u_{g}=1.91 \mathrm{~mm} / \mathrm{s}$ in the fixed bed regime, (b) $u_{g}=3.40 \mathrm{~mm} / \mathrm{s}$ in the solid-like homogeneous fluidization regime, (c) $u_{g}=5.31 \mathrm{~mm} / \mathrm{s}$ in the uniform non-bubbling fluid-like fluidization regime, and (d) $u_{g}=7.85 \mathrm{~mm} / \mathrm{s}$ in the bubbling fluidization regime. The fluidized powder is FCC I.

Figure 4. Average and Sd of the overall solid concentration at both ECT lower and upper planes against $u_{g}$. The fluidized powder is FCC I.

Figure 5. Repeated experimental results in three separate measurements for FCC I powder: (a) and (b) are ECT measurement results; (c) and (d) are pressure fluctuation results.

Figure 6. Average and NSR of the raw capacitance at ECT upper plane against $u_{g}$. The fluidized powder is FCC I.

Figure 7. Power spectral density of the overall solid concentration at ECT upper plane for different flow regimes: (a) $u_{g}=1.27 \mathrm{~mm} / \mathrm{s}$ in the fixed bed regime, (b) $u_{g}=3.40 \mathrm{~mm} / \mathrm{s}$ in the solid-like 
homogeneous fluidization regime, (c) $u_{g}=5.31 \mathrm{~mm} / \mathrm{s}$ in the uniform non-bubbling fluid-like fluidization regime, and (d) $u_{g}=7.85 \mathrm{~mm} / \mathrm{s}$ in the bubbling fluidization regime. The fluidized powder is FCC I.

Figure 8. Power spectral density of the overall solid concentration at ECT upper plane when $u_{g}$ is about 3 times the $u_{m b}$. The fluidized powder is FCC I.

Figure 9. Normalized pressure drop and Sd of the pressure drop against $u_{g}$. The fluidized powder is FCC I.

Figure 10. Sampled signals of the bed pressure drop for different flow regimes: (a) $u_{g}=1.91 \mathrm{~mm} / \mathrm{s}$ in the fixed bed regime, (b) $u_{g}=3.40 \mathrm{~mm} / \mathrm{s}$ in the solid-like homogeneous fluidization regime, (c) $u_{g}=5.31 \mathrm{~mm} / \mathrm{s}$ in the uniform non-bubbling fluid-like fluidization regime, and (d) $u_{g}=7.85 \mathrm{~mm} / \mathrm{s}$ in the bubbling fluidization regime. The fluidized powder is FCC I.

Figure 11. Power spectral density of the bed pressure drop for different flow regimes: (a) $u_{g}=1.27$ $\mathrm{mm} / \mathrm{s}$ in the fixed bed regime, (b) $u_{g}=3.40 \mathrm{~mm} / \mathrm{s}$ in the solid-like homogeneous fluidization regime, (c) $u_{g}=5.31 \mathrm{~mm} / \mathrm{s}$ in the uniform non-bubbling fluid-like fluidization regime, and (d) $u_{g}$ $=7.85 \mathrm{~mm} / \mathrm{s}$ in the bubbling fluidization regime. The fluidized powder is FCC I.

Figure 12. Time domain analysis of the overall solid concentration at ECT upper plane for (a) FCC II powder and (b) $\mathrm{Al}_{2} \mathrm{O}_{3}$ powder.

Figure 13. Sedimentation experimental and fitted results using (a) Equation 12 and (b) Equation 16 for FCC I powder. 


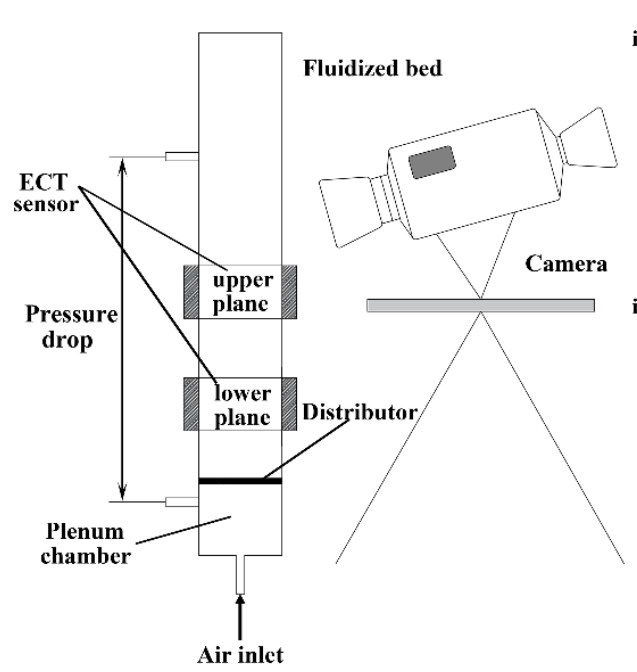

(a)

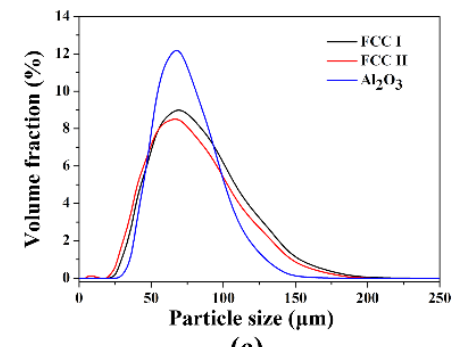

(c)

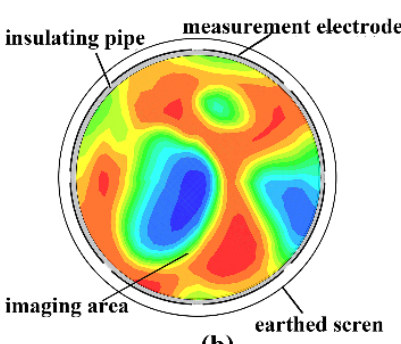

(b)

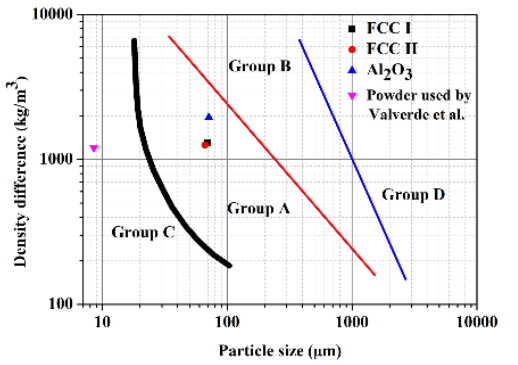

(d)

Figure 1. (a) Experimental setup. (b) ECT sensor structure. (c) Particle size distribution of the powders. (d) Situation of the powders in the Geldart diagram. 


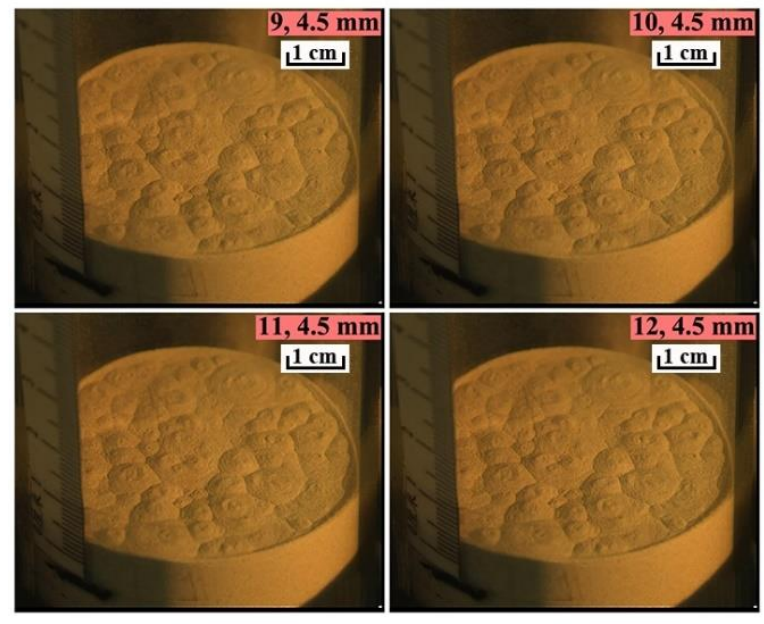

(a)

$u_{g}=1.27 \mathrm{~mm} / \mathrm{s}$, fixed bed

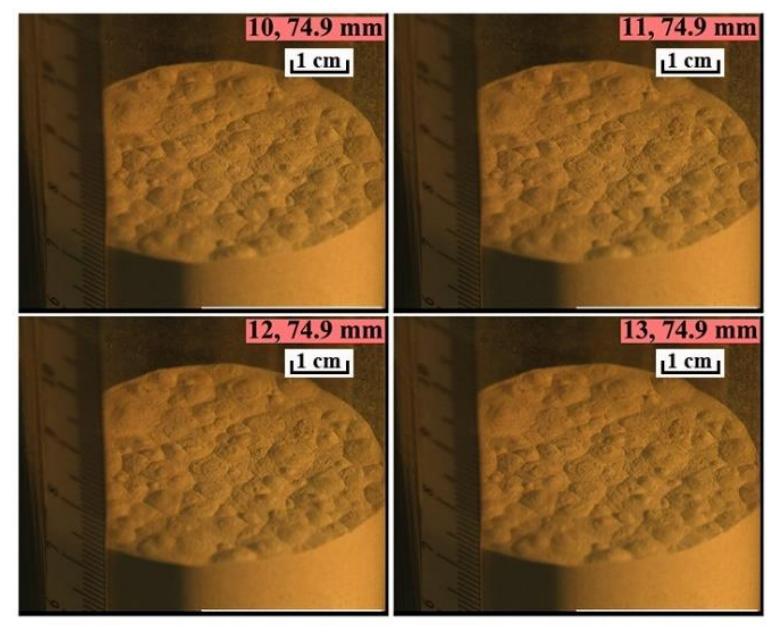

(c)

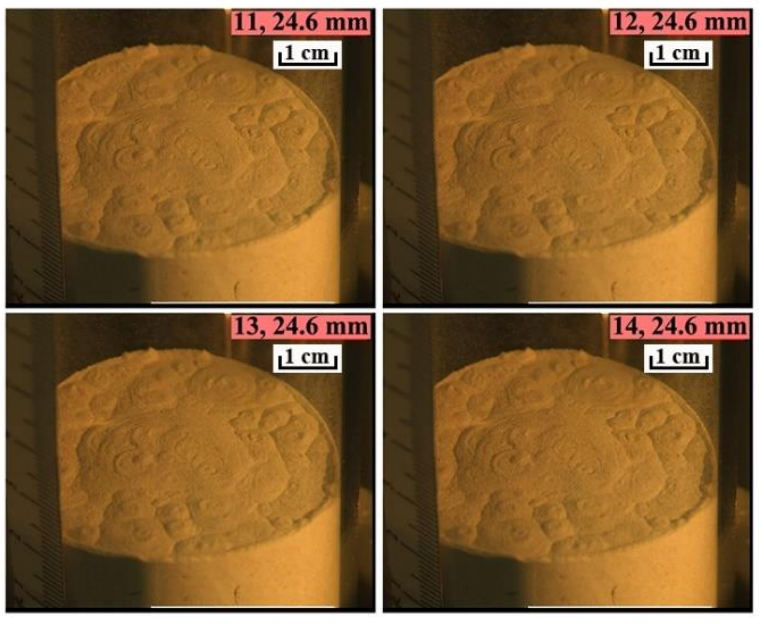

(b)

$u_{g}=3.18 \mathrm{~mm} / \mathrm{s}$, solid-like homogeneous

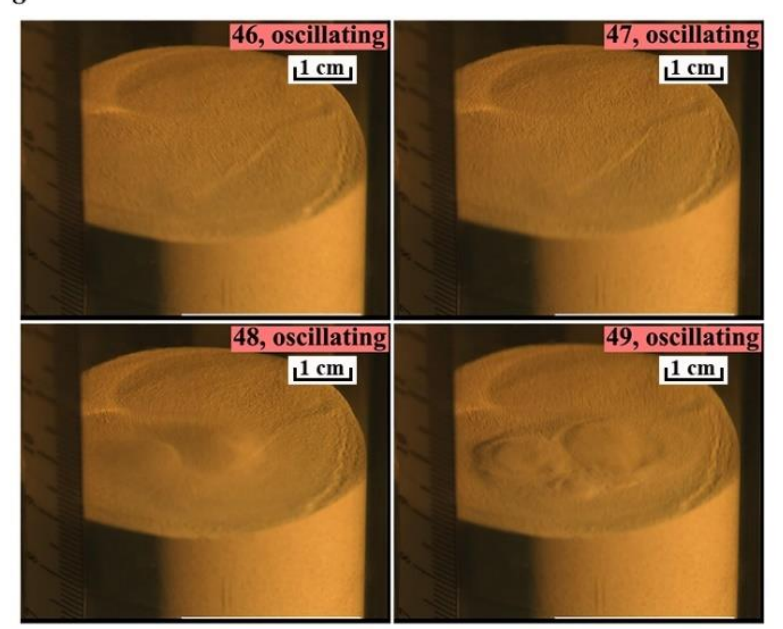

(d)

$u_{g}=5.09 \mathrm{~mm} / \mathrm{s}$, non-bubbling fluid-like $\quad u_{g}=7.85 \mathrm{~mm} / \mathrm{s}$, bubbling

Figure 2. Visual snapshots taken by the camera: (a) $u_{g}=1.27 \mathrm{~mm} / \mathrm{s}$ in the fixed bed regime, (b) $u_{g}=3.18 \mathrm{~mm} / \mathrm{s}$ in the solid-like homogeneous fluidization regime, (c) $u_{g}=5.09 \mathrm{~mm} / \mathrm{s}$ in the uniform non-bubbling fluid-like fluidization regime, and (d) $u_{g}=7.85 \mathrm{~mm} / \mathrm{s}$ in the bubbling fluidization regime. The fluidized powder is FCC I. 


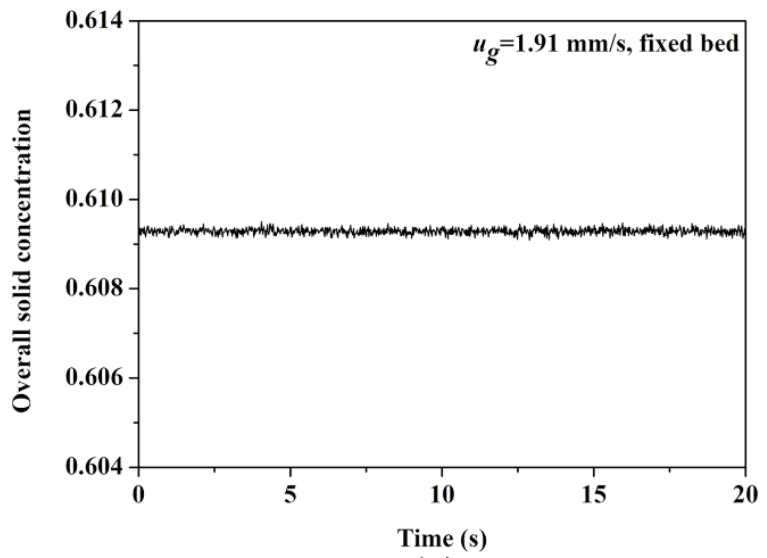

(a)

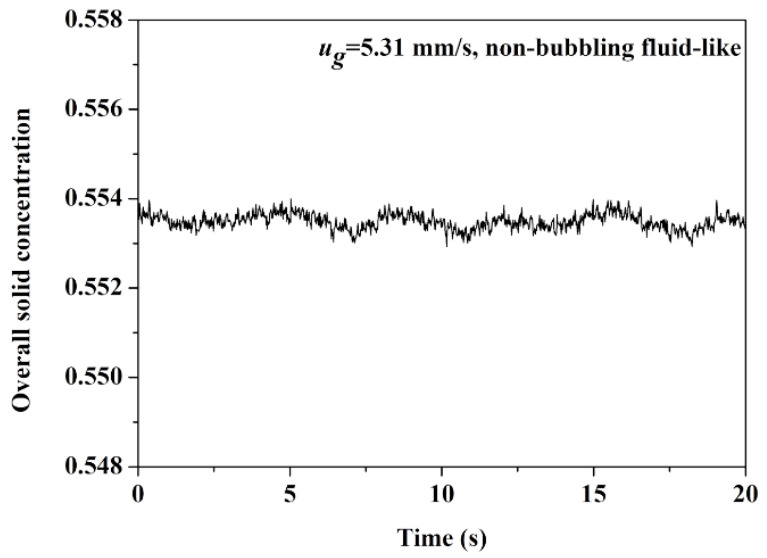

(c)

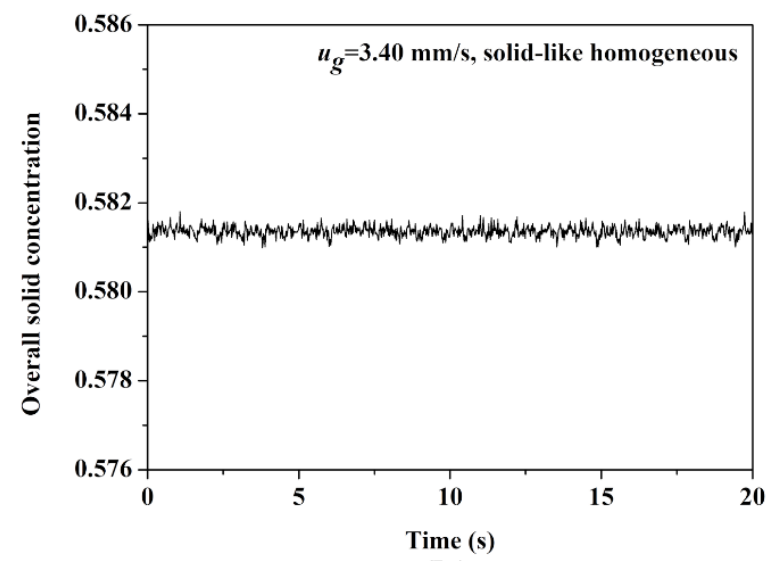

(b)

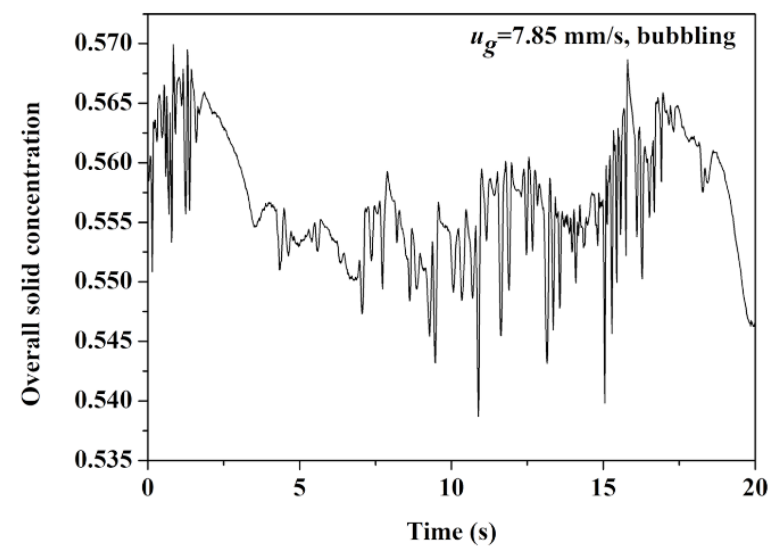

(d)

Figure 3. Sampled signals of the overall solid concentration at ECT upper plane for different flow regimes: (a) $u_{g}=1.91 \mathrm{~mm} / \mathrm{s}$ in the fixed bed regime, (b) $u_{g}=3.40 \mathrm{~mm} / \mathrm{s}$ in the solid-like homogeneous fluidization regime, (c) $u_{g}=5.31 \mathrm{~mm} / \mathrm{s}$ in the uniform non-bubbling fluid-like fluidization regime, and (d) $u_{g}=7.85 \mathrm{~mm} / \mathrm{s}$ in the bubbling fluidization regime. The fluidized powder is FCC I. 


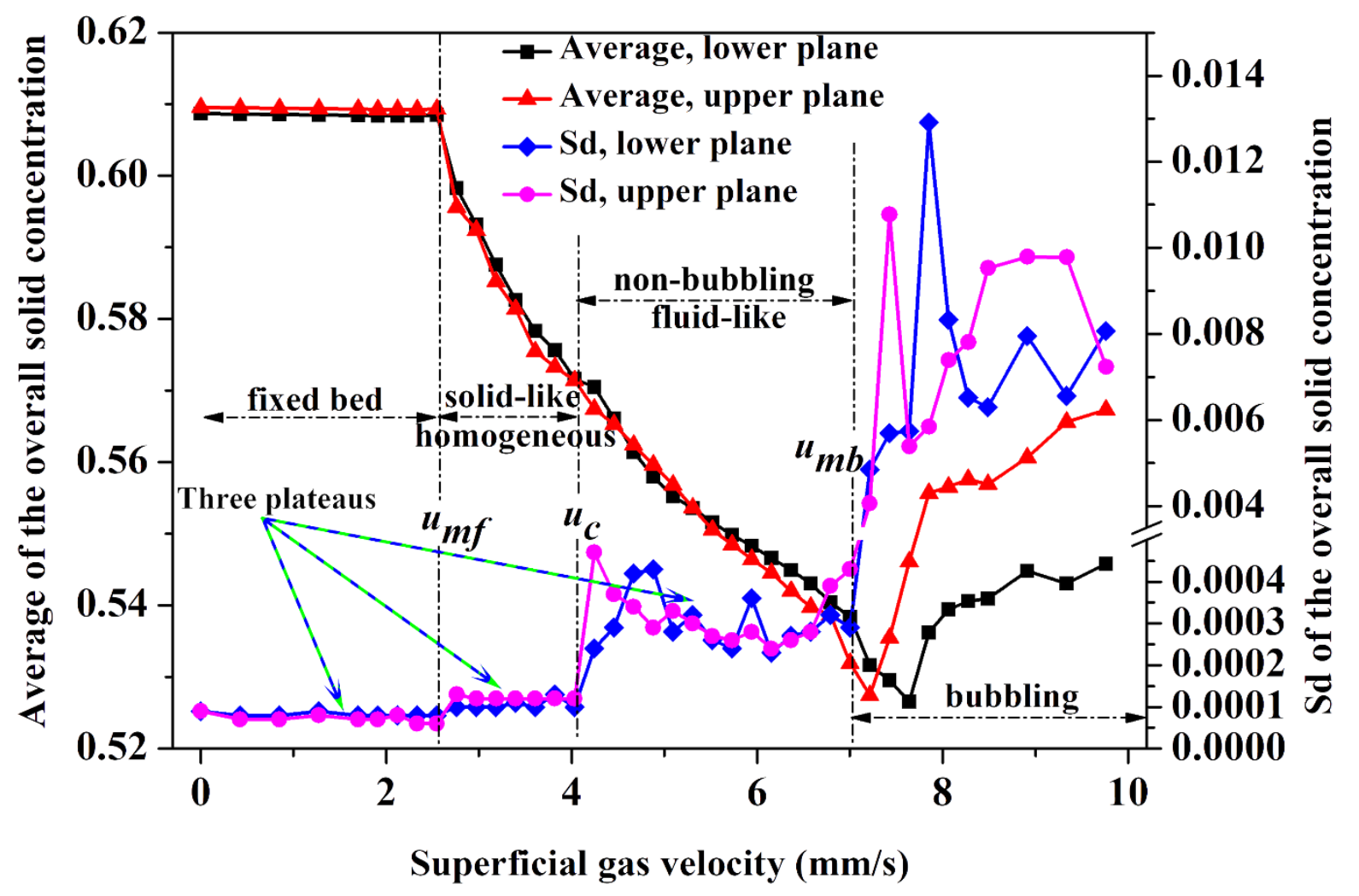

Figure 4. Average and Sd of the overall solid concentration at both ECT lower and upper planes against $u_{g}$. The fluidized powder is FCC I. 


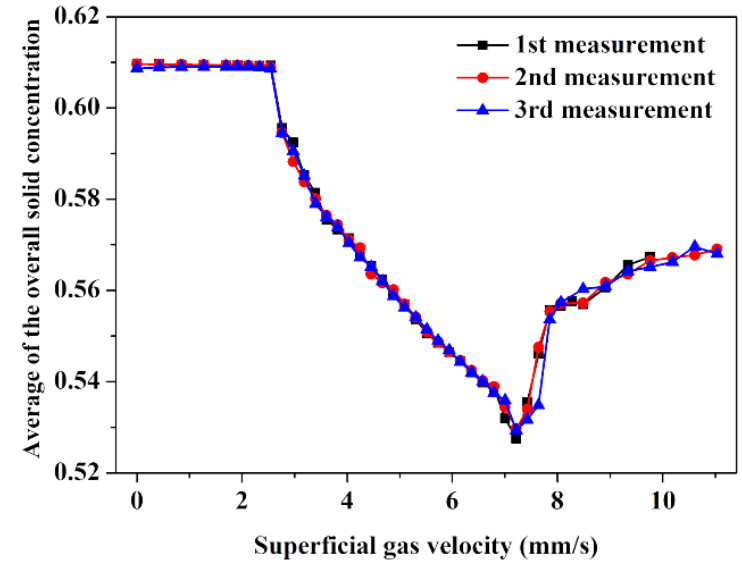

(a)

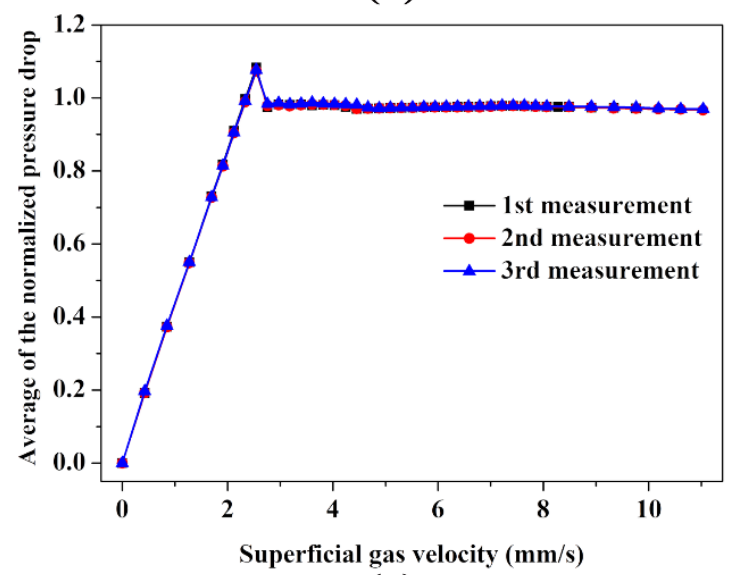

(c)

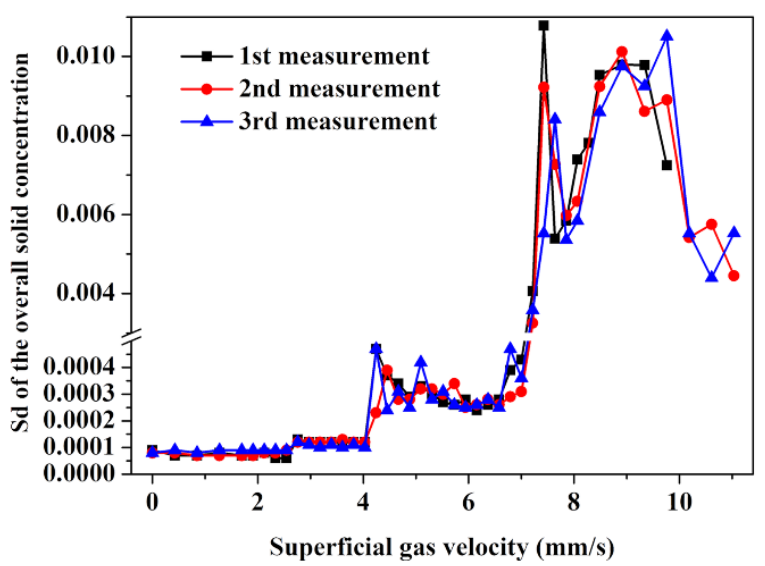

(b)

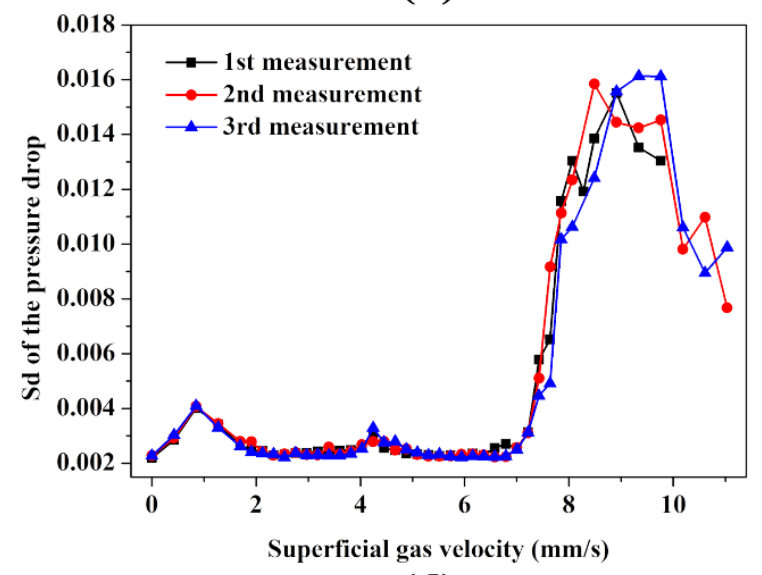

(d)

Figure 5. Repeated experimental results in three separate measurements for FCC I powder: (a) and (b) are ECT measurement results; (c) and (d) are pressure fluctuation results. 


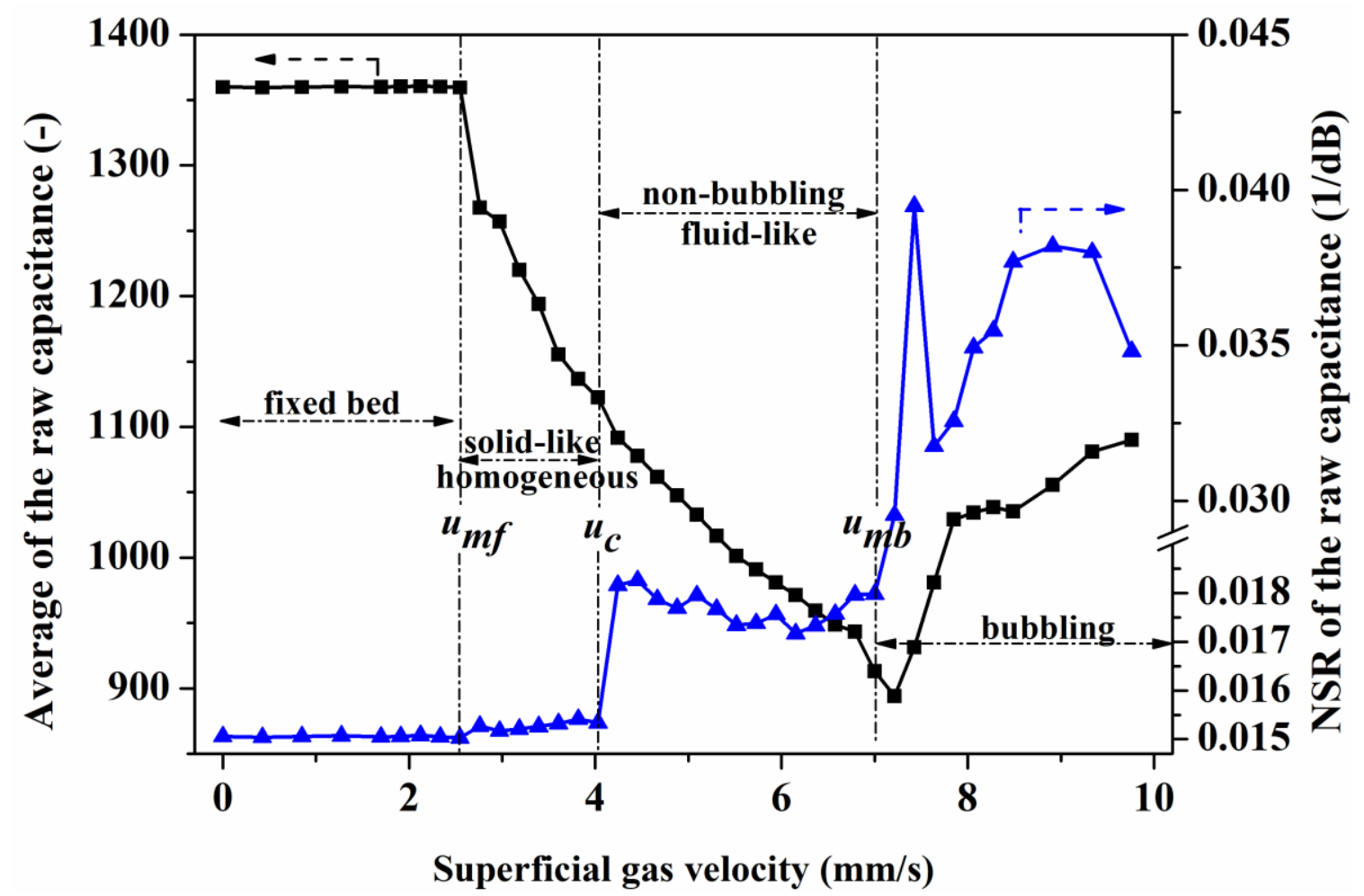

Figure 6. Average and NSR of the raw capacitance at ECT upper plane against $u_{g}$. The fluidized powder is FCC I. 


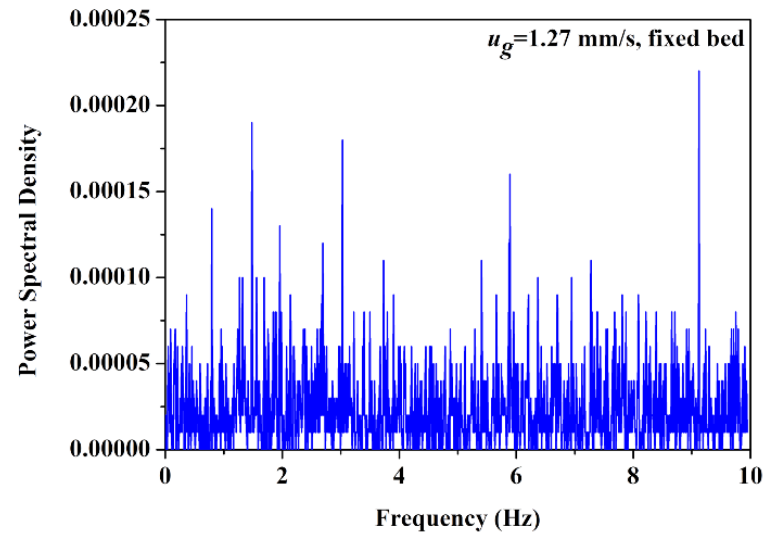

(a)

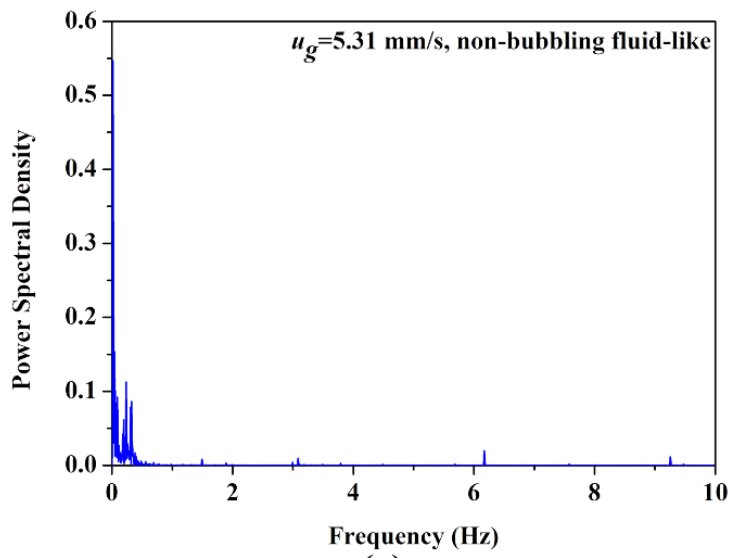

(c)

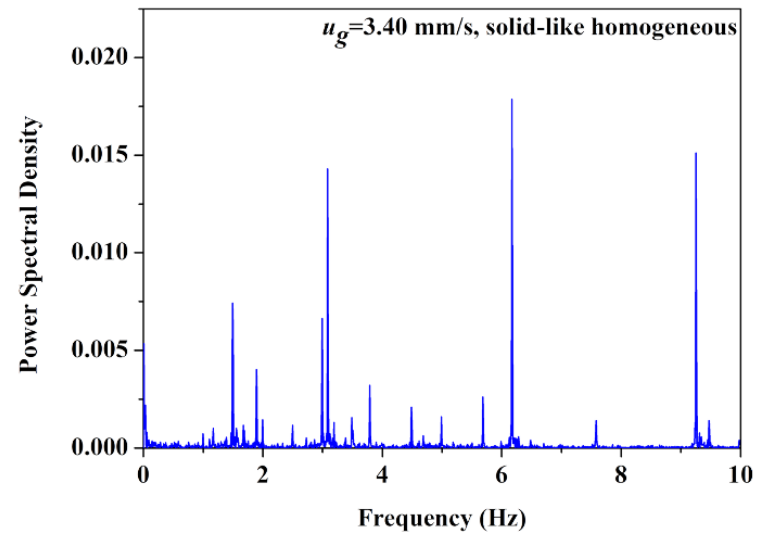

(b)

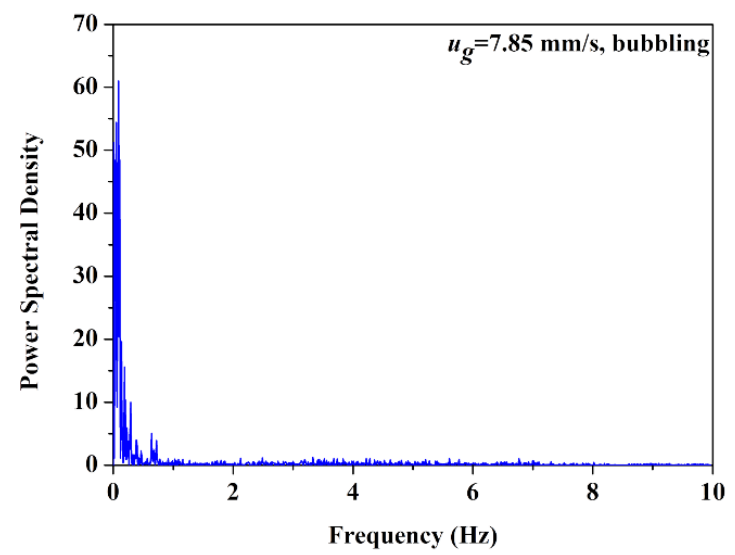

(d)

Figure 7. Power spectral density of the overall solid concentration at ECT upper plane for different flow regimes: (a) $u_{g}=1.27 \mathrm{~mm} / \mathrm{s}$ in the fixed bed regime, (b) $u_{g}=3.40 \mathrm{~mm} / \mathrm{s}$ in the solid-like homogeneous fluidization regime, (c) $u_{g}=5.31 \mathrm{~mm} / \mathrm{s}$ in the uniform non-bubbling fluid-like fluidization regime, and (d) $u_{g}=7.85 \mathrm{~mm} / \mathrm{s}$ in the bubbling fluidization regime. The fluidized powder is FCC I. 


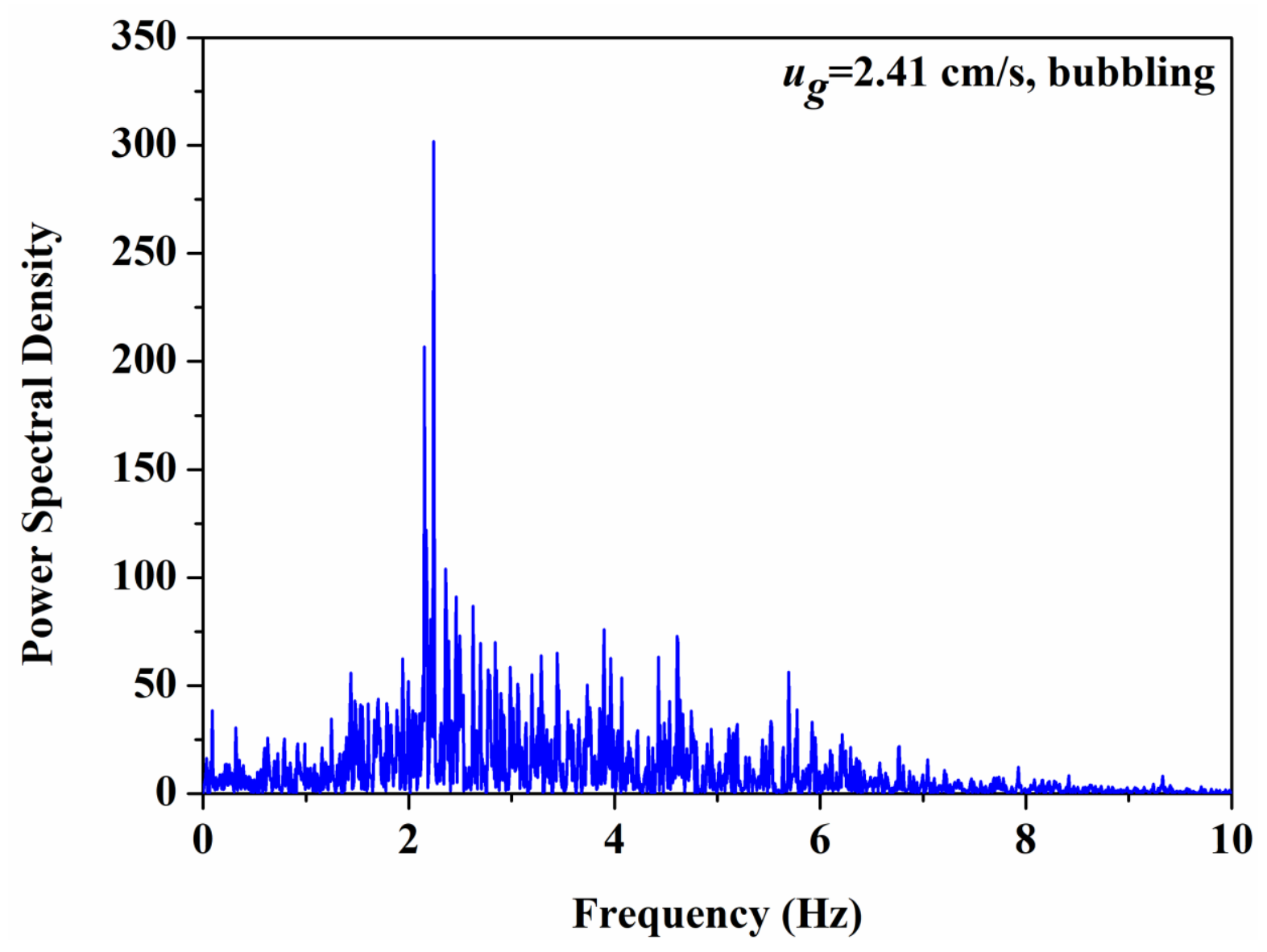

Figure 8. Power spectral density of the overall solid concentration at ECT upper plane when $u_{g}$ is about 3 times the $u_{m b}$. The fluidized powder is FCC I. 


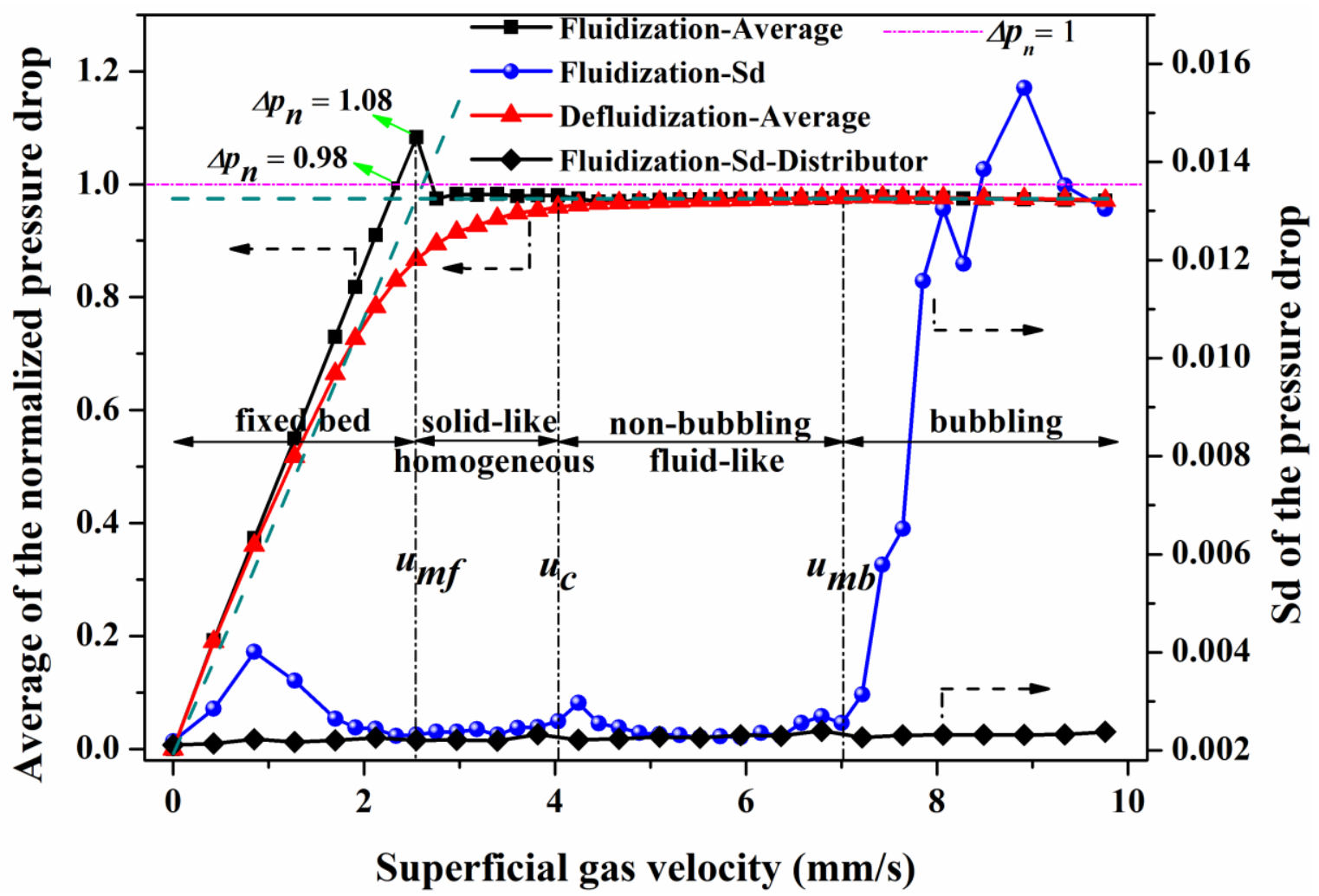

Figure 9. Normalized pressure drop and $\mathrm{Sd}$ of the pressure drop against $u_{g}$. The fluidized powder is FCC I. 


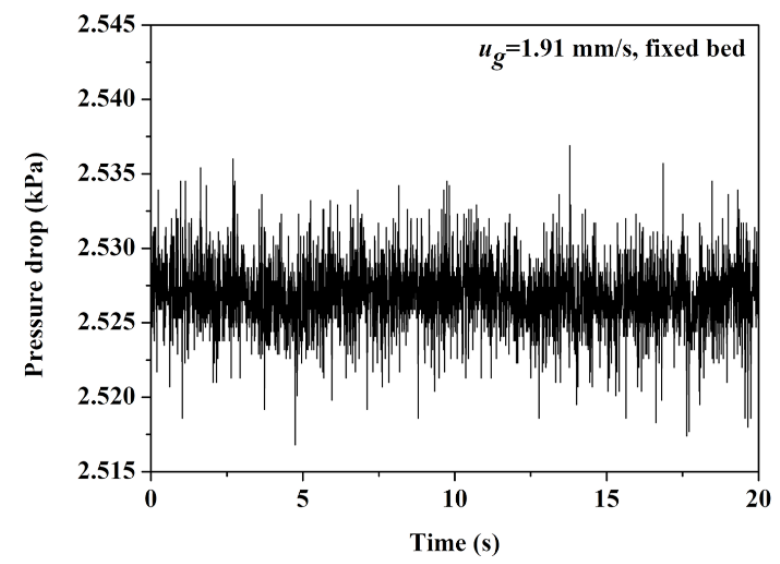

(a)

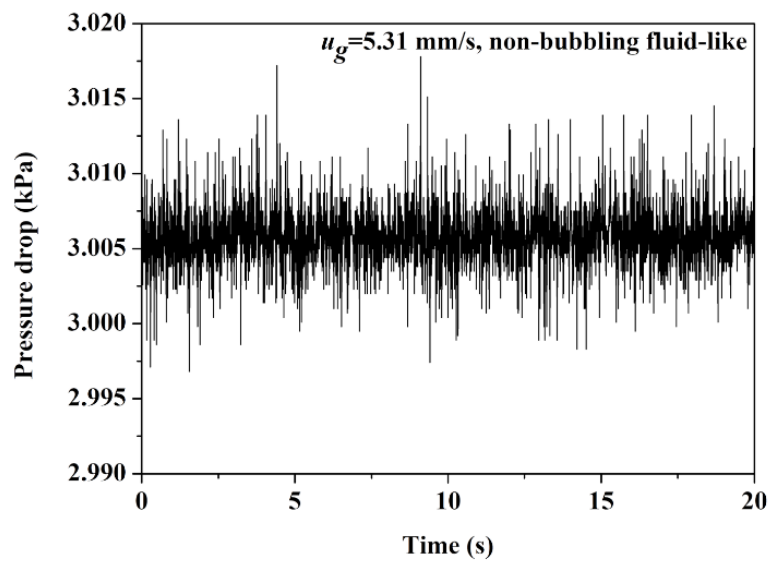

(c)

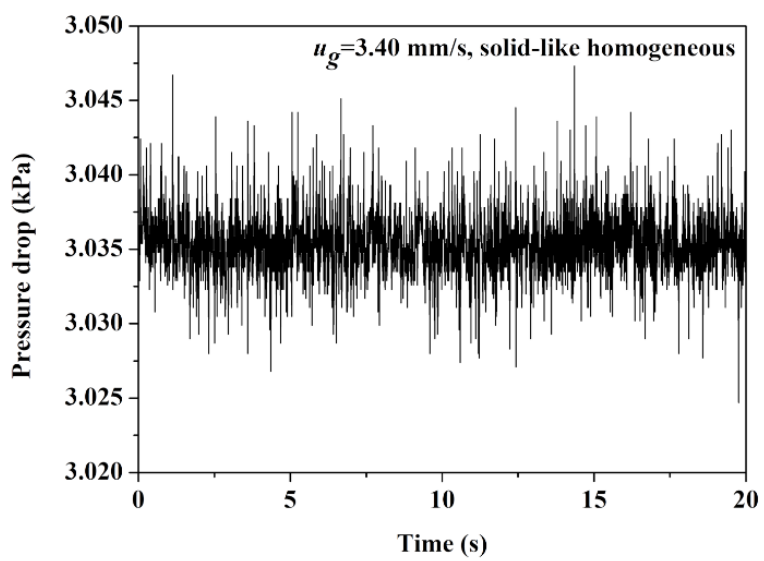

(b)

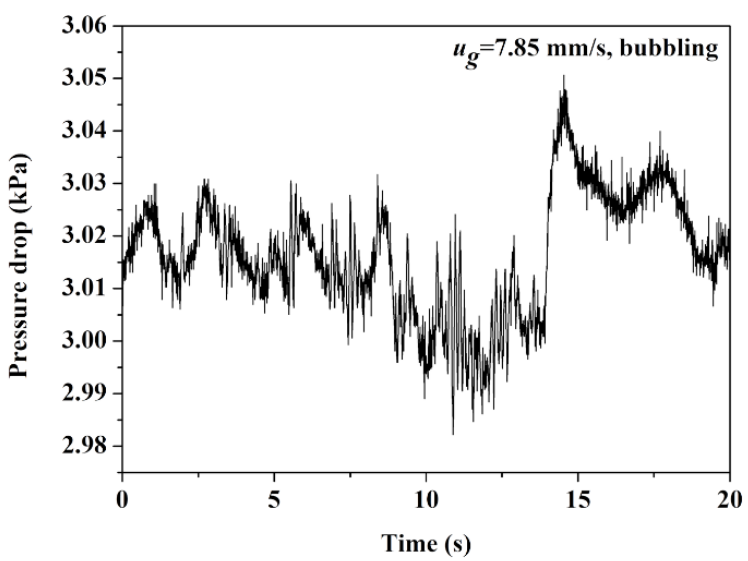

(d)

Figure 10. Sampled signals of the bed pressure drop for different flow regimes: (a) $u_{g}=1.91$ $\mathrm{mm} / \mathrm{s}$ in the fixed bed regime, (b) $u_{g}=3.40 \mathrm{~mm} / \mathrm{s}$ in the solid-like homogeneous fluidization regime, (c) $u_{g}=5.31 \mathrm{~mm} / \mathrm{s}$ in the uniform non-bubbling fluid-like fluidization regime, and (d) $u_{g}$ $=7.85 \mathrm{~mm} / \mathrm{s}$ in the bubbling fluidization regime. The fluidized powder is FCC I. 


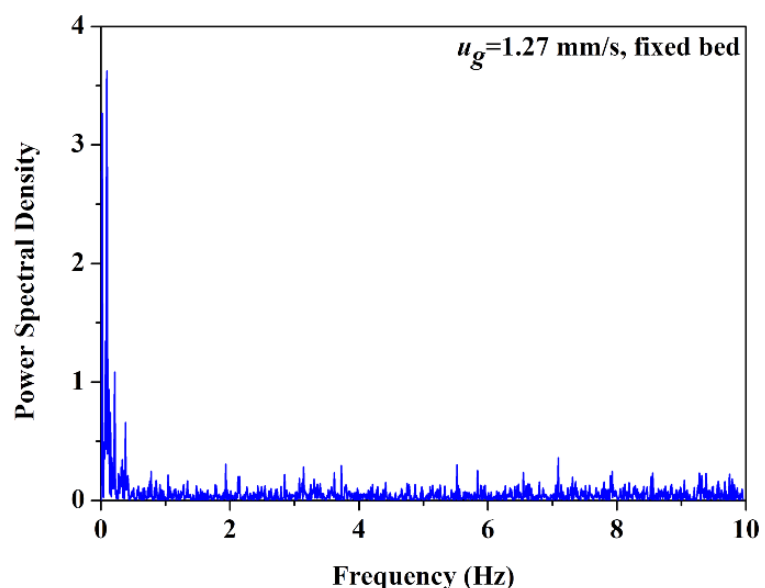

(a)

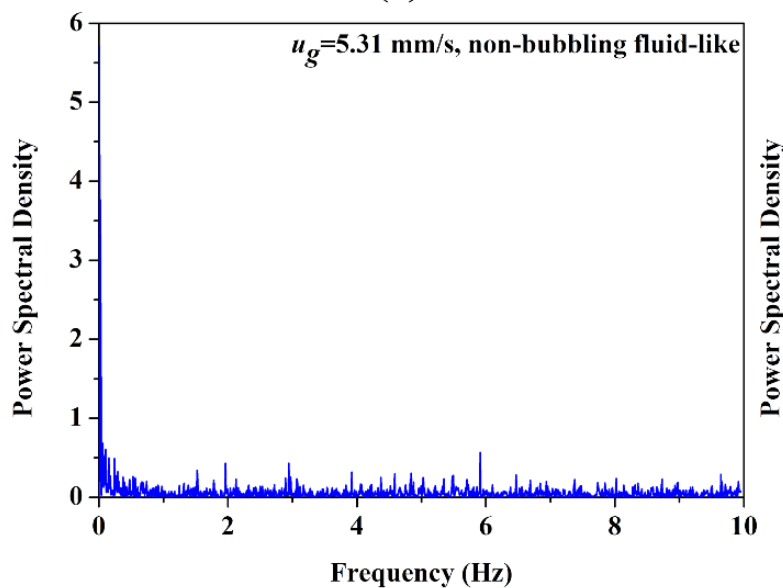

(c)

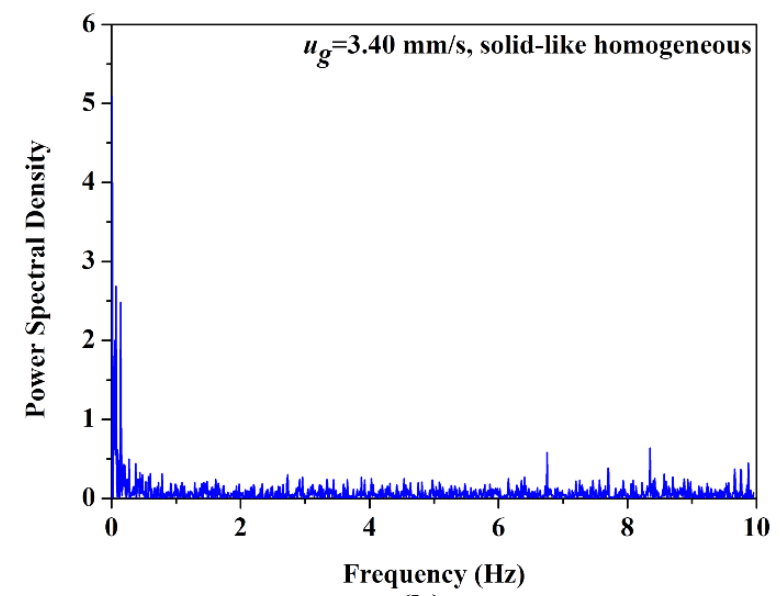

(b)

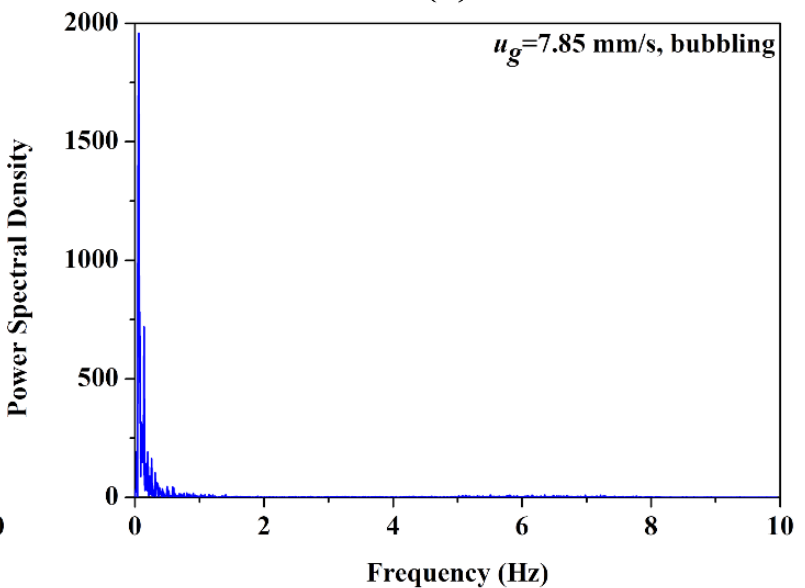

(d)

Figure 11. Power spectral density of the bed pressure drop for different flow regimes: (a) $u_{g}=$ $1.27 \mathrm{~mm} / \mathrm{s}$ in the fixed bed regime, (b) $u_{g}=3.40 \mathrm{~mm} / \mathrm{s}$ in the solid-like homogeneous fluidization regime, (c) $u_{g}=5.31 \mathrm{~mm} / \mathrm{s}$ in the uniform non-bubbling fluid-like fluidization regime, and (d) $u_{g}$ $=7.85 \mathrm{~mm} / \mathrm{s}$ in the bubbling fluidization regime. The fluidized powder is FCC I. 


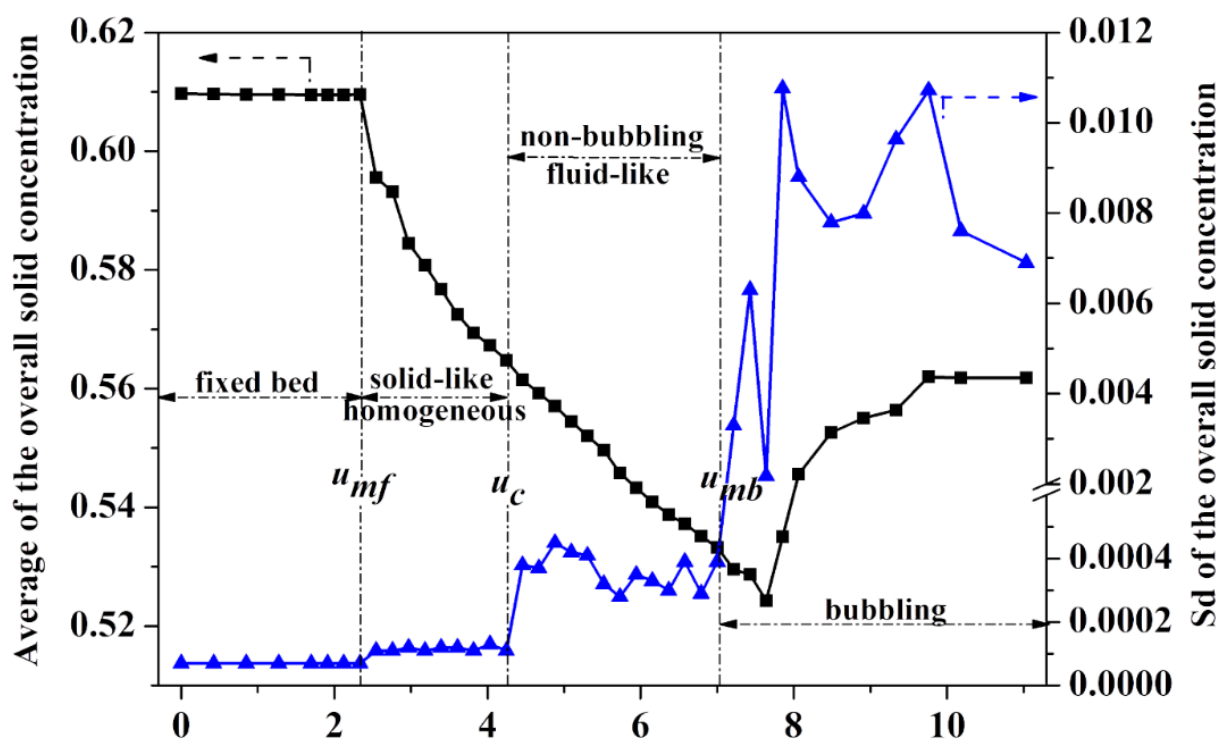

Superficial gas velocity $(\mathrm{mm} / \mathrm{s})$

(a)

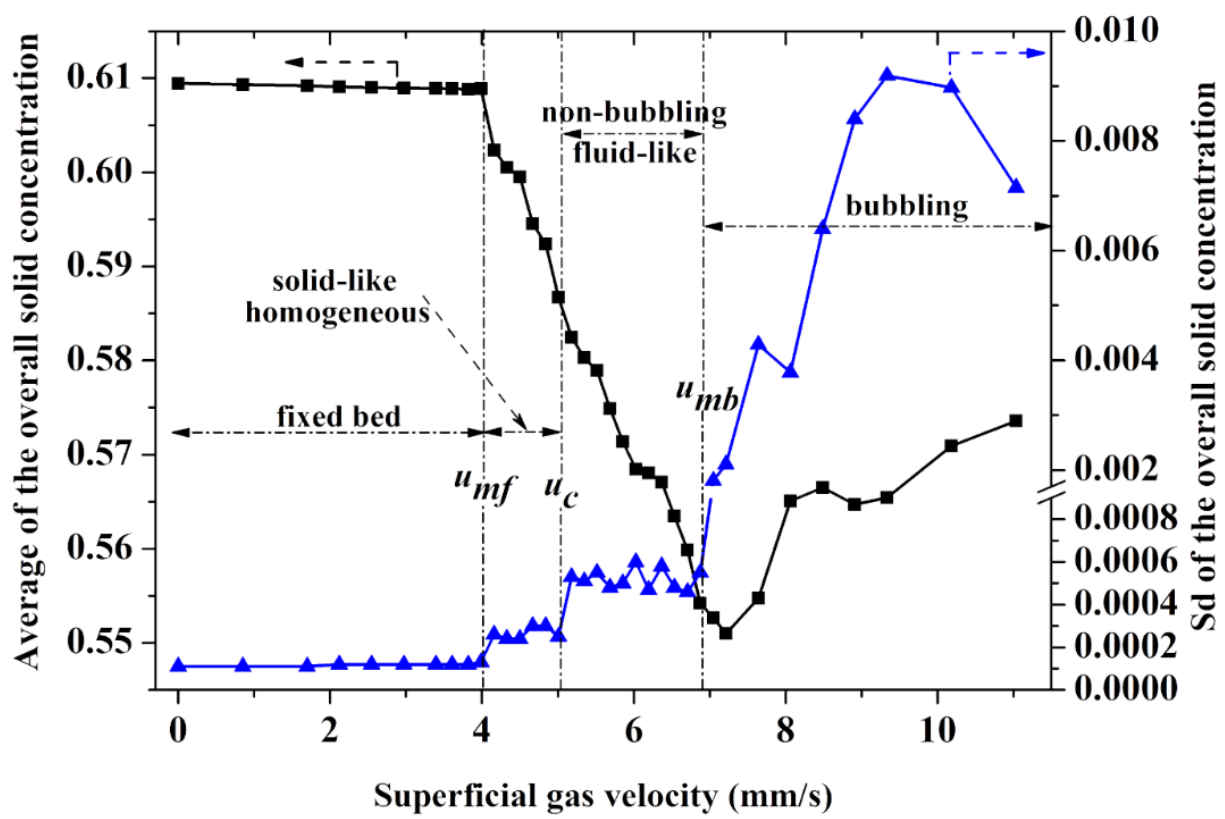

(b)

Figure 12. Time domain analysis of the overall solid concentration at ECT upper plane for (a)

FCC II powder and (b) $\mathrm{Al}_{2} \mathrm{O}_{3}$ powder. 


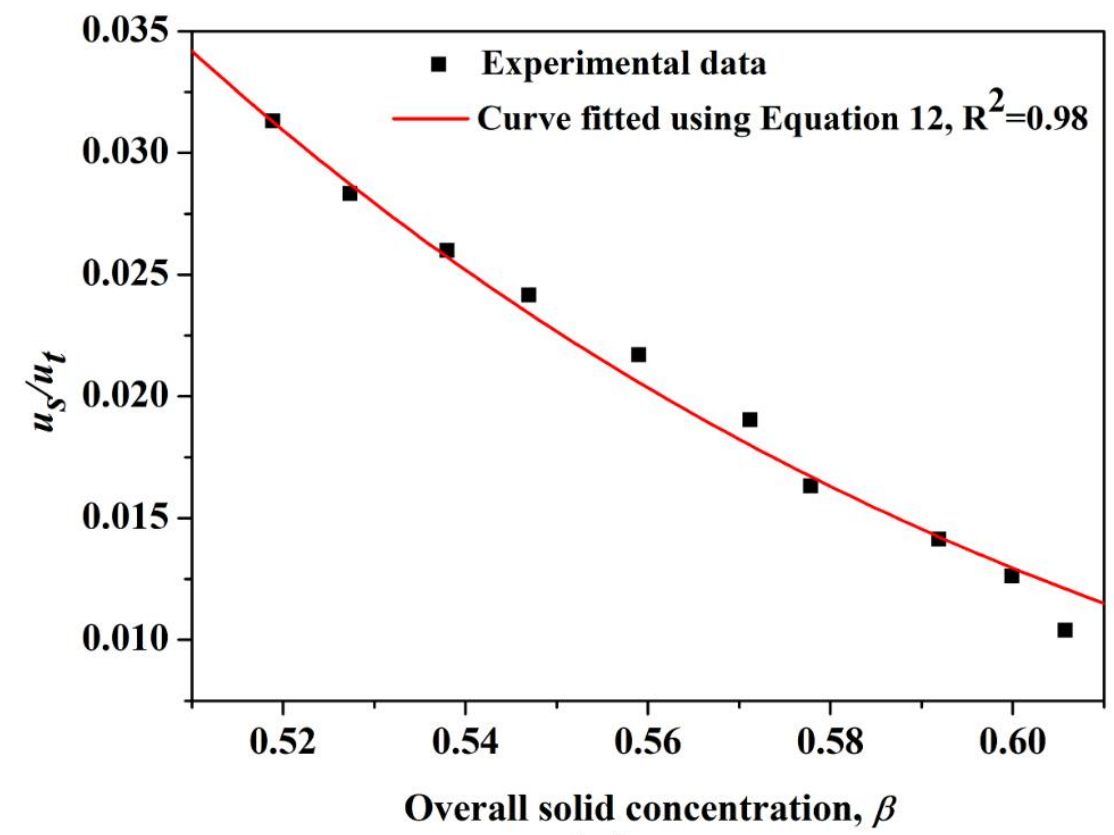

(a)

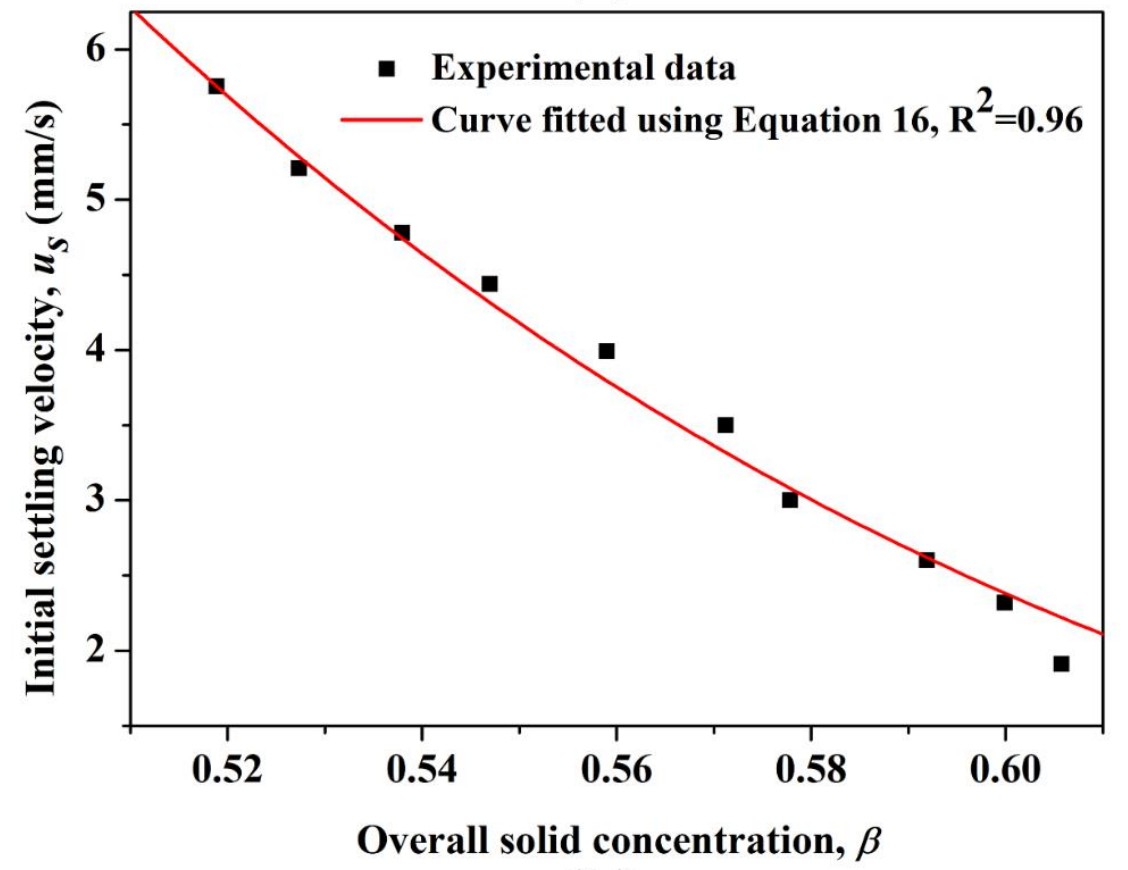

(b)

Figure 13. Sedimentation experimental and fitted results using (a) Equation 12 and (b) Equation 16 for FCC I powder. 


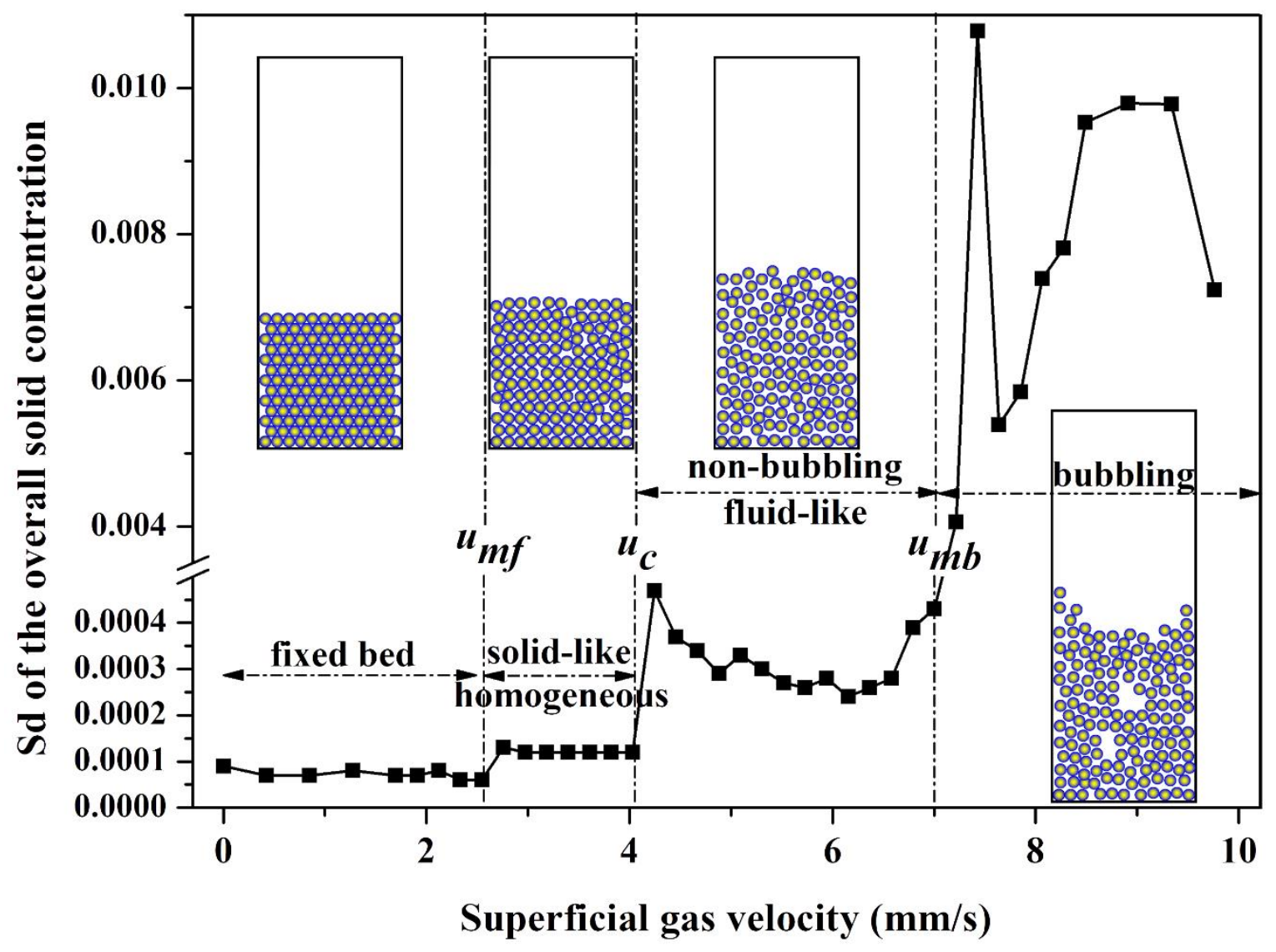

Abstract Graphic. For Table of Contents Only. 